\title{
The Gaia-ESO Survey: Detailed abundances in the metal-poor globular cluster NGC 4372
}

I. San Roman ${ }^{1,2}$, C. Muñoz ${ }^{1}$, D. Geisler ${ }^{1}$, S. Villanova ${ }^{1}$, N. Kacharov ${ }^{3}$, A. Koch $^{3}$, G. Carraro ${ }^{4}$, G. Tautvaišiene ${ }^{5}$, A. Vallenari ${ }^{6}$, E. J. Alfaro ${ }^{7}$, T. Bensby ${ }^{8}$, E. Flaccomio ${ }^{9}$, P. Francois ${ }^{10}$, A. J. Korn ${ }^{11}$, E. Pancino ${ }^{12,13}$, A. Recio-Blanco ${ }^{14}$, R. Smiljanic ${ }^{15}$, M. Bergemann ${ }^{16}$, M. T. Costado ${ }^{7}$, F. Damiani ${ }^{9}$, U. Heiter ${ }^{11}$, A. Hourihane ${ }^{16}$, P. Jofré ${ }^{16}$, C. Lardo $^{17}$, P. de Laverny ${ }^{14}$, T. Masseron ${ }^{16}$, L. Morbidelli ${ }^{18}$, L. Sbordone ${ }^{19,20}$, S. G. Sousa ${ }^{21,22}$, C. C. Worley ${ }^{16}$, and S. Zaggia ${ }^{6}$

${ }^{1}$ Departamento de Astronomía, Casilla 160-C, Universidad de Concepción, Concepción, Chile e-mail: isanroman@cefca.es

2 Centro de Estudios de Física del Cosmos de Aragón (CEFCA), Plaza San Juan 1, 44001 Teruel, Spain

${ }^{3}$ Landessternwarte, Zentrum für Astronomie der Universität Heidelberg, Königstuhl 12, 69117 Heidelberg, Germany

${ }^{4}$ ESO, Alonso de Cordova 3107, 19001 Santiago de Chile, Chile

5 Institute of Theoretical Physics and Astronomy, Vilnius University, Goštauto 12, 01108 Vilnius, Lithuania

${ }^{6}$ INAF-Padova Observatory, Vicolo dell'Osservatorio 5, 35122 Padova, Italy

7 Instituto de Astrofísica de Andalucía-CSIC, Apdo. 3004, 18080 Granada, Spain

${ }^{8}$ Lund Observatory, Department of Astronomy and Theoretical Physics, Box 43, 22100 Lund, Sweden

9 INAF-Osservatorio Astronomico di Palermo, Piazza del Parlamento 1, 90134 Palermo, Italy

10 GEPI, Observatoire de Paris, CNRS, Université Paris Diderot, 5 place Jules Janssen, 92190 Meudon, France

11 Department of Physics and Astronomy, Uppsala University, Box 516, 75120 Uppsala, Sweden

12 INAF-Osservatorio Astronomico di Bologna, via Ranzani 1, 40127 Bologna, Italy

13 ASI Science Data Center, via del Politecnico SNC, 00133 Roma, Italy

14 Laboratoire Lagrange (UMR 7293), Université de Nice Sophia Antipolis, CNRS, Observatoire de la Côte d'Azur, CS 34229, 06304 Nice Cedex 4, France

15 Department for Astrophysics, Nicolaus Copernicus Astronomical Center, ul. Rabiańska 8, 87-100 Toruń, Poland

16 Institute of Astronomy, University of Cambridge, Madingley Road, Cambridge CB3 OHA, UK

17 Astrophysics Research Institute, Liverpool John Moores University, 146 Brownlow Hill, Liverpool L3 5RF, UK

18 INAF-Osservatorio Astrofisico di Arcetri, Largo E. Fermi 5, 50125 Florence, Italy

19 Millennium Institute of Astrophysics, Av. Vicuña Mackenna 4860, 782-0436 Macul, Santiago, Chile

20 Pontificia Universidad Católica de Chile, Av. Vicuña Mackenna 4860, 782-0436 Macul, Santiago, Chile

21 Centro de Astrofísica, Universidade do Porto, Rua das Estrelas, 4150-762 Porto, Portugal

22 Departamento de Física e Astronomia, Faculdade de Ciências, Universidade do Porto, Rua do Campo Alegre, 4169-007 Porto, Portugal

${ }^{23}$ Institute of Astronomy, University of Cambridge, Madingley Road, Cambridge CB3 OHA, UK

Received 23 January 2015 / Accepted 13 April 2015

\section{ABSTRACT}

We present the abundance analysis for a sample of 7 red giant branch stars in the metal-poor globular cluster NGC 4372 based on UVES spectra acquired as part of the Gaia-ESO Survey. This is the first extensive study of this cluster from high-resolution spectroscopy. We derive abundances of $\mathrm{O}, \mathrm{Na}, \mathrm{Mg}, \mathrm{Al}, \mathrm{Si}, \mathrm{Ca}, \mathrm{Sc}, \mathrm{Ti}, \mathrm{Fe}, \mathrm{Cr}, \mathrm{Ni}, \mathrm{Y}, \mathrm{Ba}$, and $\mathrm{La}$. We find a metallicity of $[\mathrm{Fe} / \mathrm{H}]=$ $-2.19 \pm 0.03$ and find no evidence of any metallicity spread. This metallicity makes NGC 4372 one of the most metal-poor Galactic globular clusters. We also find an $\alpha$-enhancement typical of halo globular clusters at this metallicity. Significant spreads are observed in the abundances of light elements. In particular, we find a $\mathrm{Na}-\mathrm{O}$ anticorrelation. Abundances of $\mathrm{O}$ are relatively high compared with other globular clusters. This could indicate that NGC 4372 was formed in an environment with high O for its metallicity. A $\mathrm{Mg}-\mathrm{Al}$ spread is also present that spans a range of more than 0.5 dex in $\mathrm{Al}$ abundances. $\mathrm{Na}$ is correlated with $\mathrm{Al}$ and $\mathrm{Mg}$ abundances at a lower significance level. This pattern suggests that the Mg-Al burning cycle is active. This behavior can also be seen in giant stars of other massive, metal-poor clusters. A relation between light and heavy $s$-process elements has been identified.

Key words. globular clusters: individual: NGC 4372 - stars: abundances

\section{Introduction}

Globular clusters (GCs) provide an exceptional laboratory for studying the star formation history of a galaxy because they are powerful tracers of the various components (halo, thick disk, and bulge). Moreover, the ages, abundances, and kinematics of these objects represent the fossil record of the galaxy formation process, since they are among the oldest objects in the Universe. Starting several decades ago, both spectroscopy and photometry (e.g. Piotto et al. 2007; Gratton et al. 2012) have shown that GCs are much more complex than previously imagined, in particular with the almost ubiquitous discovery of multiple populations. 
None of the scenarios proposed to date can fully account for the abundance trends observed in Galactic globular clusters (GGCs). Detailed chemical studies of large samples of GGCs are required to uncover the evolution of these objects. Providing high-quality data on more GCs is clearly needed to strengthen any conclusion. We present here results of the first-ever high-resolution spectroscopic analysis of the globular cluster NGC 4372 based on observations from the Gaia-ESO survey (GES, Gilmore et al. 2012; Randich \& Gilmore 2012).

The GES is a public spectroscopic survey using the highresolution multi-object spectrograph FLAMES on the Very Large Telescope. Targeting more than $10^{5}$ stars, GES covers all the major components of the Milky Way from halo to star forming regions, thereby providing a homogeneous overview of the distribution of kinematics and elemental abundances. The survey also maps a significant sample of more than 80 open clusters, covering all the accessible cluster ages and masses.

Ensuring that GES has a maximum legacy impact is a key priority. Thus the survey has identified a suitable set of objects and fields for calibrating the data in terms of astrophysical parameters and abundance ratios (Pancino et al. 2012). By including objects and fields that are a) well-studied in the literature; b) in common with other large spectroscopic surveys; and/or c) have extremely well measured properties, the results can be put into context, compared with other surveys, and when available, combined with different data sets. The GES includes GGCs as calibrators, adding some relatively unstudied clusters to the ones already present in the ESO archive. To maximize the scientific output from the calibrator data set, a good balance of mandatory, known, and new or interesting GGCs has been included in the target selection. Within this context, this study focuses on the chemical abundances of NGC 4372. With only limited spectroscopic data, nothing is known about any possible abundance variations of this metal-poor halo cluster. We present here an abundance analysis of a large number of elements (e.g., light elements, $\alpha$ elements, iron peak elements, and neutron-capture elements) and analyze its stellar population.

The vast majority of old GGCs studied in detail to date, with the possible exception of Rup 106 (Villanova et al. 2013), show the chemical signatures of hosting (at least) two stellar populations. Several other cases like Pal 12 (Cohen 2004), Ter 7 (Tautvaišienè et al. 2004; Sbordone et al. 2005), or Ter 8 (Carretta et al. 2014) have been proposed as potential singlepopulation globular clusters, but the small sample investigated does not allow for clear conclusions. We also note that all these objects except for Rup 106 are associated with the Sgr dwarf galaxy. There has been much improvement in the study of multiple populations within GGCs. The main evidence for this complexity comes from the chemical inhomogeneities that in most cases are limited to light elements. Light elements like Li, C, N, $\mathrm{O}, \mathrm{Na}, \mathrm{Mg}$, and $\mathrm{Al}$, are known to (anti-)correlate. The most outstanding signature is the Na-O anticorrelation, detected so far in every GC study (Carretta et al. 2009a,b), again with the likely exception of Rup 106. These anomalies have also been observed in old, massive extragalactic GCs in Fornax (Letarte et al. 2006) and in the Large Magellanic Cloud (LMC; Mucciarelli et al. 2009), but not in intermediate-age LMC clusters (Mucciarelli et al. 2014). A similar feature, often observed in some GCs, but not all, is the anticorrelation between $\mathrm{Al}$ and $\mathrm{Mg}$ (Gratton et al. 2001; Carretta et al. 2009b). It is important to mention that these abundance trends are not seen in the halo field stars.

This spectroscopic evidence has been interpreted as the signature of material processed during H-burning by high temperature proton-capture reactions (such as the $\mathrm{Ne}-\mathrm{Na}$ and
Mg-Al cycles). Several theoretical models have been proposed to describe the formation and early evolution of GCs (D'Ercole et al. 2008). The preferred explanation involves a self-enrichment scenario, within which two subsequent generations of stars co-exist in globular clusters and where the second is formed from gas polluted by processed material produced by massive stars of the first (Caloi \& D'Antona 2011). Several sources of processed ejecta have been proposed: the slow winds of intermediate-mass asymptotic giant branch (AGB) stars (D’Antona et al. 2002), fast-rotating massive stars (Decressin et al. 2007), and massive binaries (de Mink et al. 2009). A recent attractive alternative has been proposed by Bastian et al. (2013) that implies only a single burst of star formation. They postulate that the polluted gas concentrates in the center and is acquired by low-mass stars of the first (and only) generation via disk accretion, while they are in the fully convective phase of the premain sequence. This scenario has the distinct advantage of not requiring that a very high percentage of the original cluster population has been lost. In addition, variations in heavier elements have also been found in some massive GCs, such as $\omega$ Centauri (Marino et al. 2011), M54 (Carretta et al. 2010b), M22 (Marino et al. 2009), NGC 1851 (Carretta et al. 2011), Terzan5 (Ferraro et al. 2009), NGC 2419 (Cohen et al. 2010), M2 (Lardo et al. 2013; Yong et al. 2014), and M75 (Kacharov et al. 2013). They are generally thought to be the vestige of more massive primitive dwarf galaxies that merged with the Galaxy. Therefore, they have important implications for the hierarchical merging scenario of galaxy formation (Joo \& Lee 2013). In this paper, we use the widely accepted terminology of first and second generation of stars, referring to the unpolluted and polluted populations regardless of the formation scenario.

NGC 4372 is an old and very metal-poor globular cluster that despite its low degree of central concentration, has received little attention, mostly due to reddening problems. It is located close to the Galactic disk and in a dusty area of the Southern Musca Constellation $(\mathrm{RA}=12: 25: 45.40$, Dec $=-72: 39: 32.4$; $l=300.99^{\circ}, b=-9.88^{\circ}$ Harris 1996, 2010 edition). At a Galactocentric distance of $R_{\mathrm{gc}}=7.1 \mathrm{Kpc}$, NGC 4372 is listed in the Harris catalog as having a low metallicity of $[\mathrm{Fe} / \mathrm{H}]=-2.17$ and a fairly high reddening of $E(B-V)=0.39$, which makes it difficult to see. In addition, NGC 4372 has been claimed to be dynamically associated with NGC 2808 (Casetti-Dinescu et al. 2007).

Early photometric studies of this cluster (Hartwick \& Hesser 1973; Alcaino 1974; Brocato et al. 1996) already revealed a color-magnitude diagram (CMD) characteristic of very metalpoor clusters with a well-defined horizontal branch that extends far to the blue and with large and variable absorption. Alcaino et al. (1991) present BVRI CCD photometry in two overlapping fields. By comparison with theoretical isochrones, they derive an age of $15 \pm 4$ Gyr. In a more recent study, Rosenberg et al. (2000) present a homogeneous photometric catalog of 39 GGCs in the southern hemisphere. For NGC 4372 they report a foreground reddening of $E(B-V)=0.42$ and a horizontal branch (HB) level of $V_{\mathrm{HB}}=15.30$ with a $\mathrm{HB}$ ratio $(B-R) /(B+V+R)=1.00$. They explain the broadening of the CMD sequences as the consequence of the high differential reddening probably due to the nearby Coal-sack Nebulae. A dereddened, narrower CMD has recently been constructed by Kacharov et al. (2014).

Spectroscopic studies of NGC 4372 are limited to mediumresolution spectra. Using the near-infrared Ca II triplet, Geisler et al. (1995) determine a mean metal abundance of $[\mathrm{Fe} / \mathrm{H}]=$ $-2.10 \pm 0.04$ from 11 giant stars. They also report a mean heliocentric radial velocity of $v_{\mathrm{rad}}=73.2 \pm 1.4 \mathrm{~km} \mathrm{~s}^{-1}$. Using a similar 
technique, Rutledge et al. (1997) estimate a value of $[\mathrm{Fe} / \mathrm{H}]=$ $-2.03 \pm 0.03$ on the Zinn \& West $(1984$, ZW84) scale and a value of $[\mathrm{Fe} / \mathrm{H}]=-1.88 \pm 0.05$ on the Carretta \& Gratton (1997, CG97) scale. A more updated value of $[\mathrm{Fe} / \mathrm{H}]=-2.19 \pm 0.08$ is provided by Carretta et al. (2009a). This value is determined from the previously mentioned Ca II triplet data but based on a more accurate metallicity scale. No further chemical study has been performed.

In this paper, we present the first extensive study of NGC 4372 from high-resolution spectroscopy. We perform an abundance analysis of a large number of elements and analyze the stellar population of the cluster. This paper is organized as follows. Section 2 describes the observations and data reduction, and Sect. 3 describes the methodology used to obtain the atmospheric parameters and chemical abundances. Section 4 presents our results including analysis of iron-peak elements, $\alpha$ elements, and any (anti)correlation. Finally, Sect. 5 presents a summary of our main results.

\section{Observations and data reduction}

The present work is based on the data collected by the GES from the beginning of the survey up to the end of June 2013. These observations are referred to as gesiDR2iDR3 and are available inside the GES collaboration. As explained in Gilmore et al. (2012), the GES consortium is structured in several working groups, WGs, that have specific duties from target selection and data reduction to delivery of science data. The data reduction was performed using a workflow specifically developed for and by GES that runs the ESO public pipeline (Modigliani et al. 2004; Modigliani \& Larsen 2012). The pipeline was optimized to reduce GES data, and it automatically performs sky subtraction, barycentric correction, and normalization. In addition, the pipeline calculates radial velocities and a first guess of the rotational velocities. A quality control was performed using the output parameters from the ESO pipeline, by a visual inspection of the spectra, and by the analysis of the signal-to-noise ratio of the spectra. A detailed description of the data reduction methods can be found in Sacco et al. (2014).

The present study only makes use of the UVES observations. While a small number of fibers can be dedicated to UVES, their spectra have higher resolution than GIRAFFE and, just as important, have ten times wider spectral coverage. For this reason, UVES data are crucial for studies of precise chemical abundances of a large number of elements. As explained in Sect. 3, this paper only makes use of Concepcion Node atmospheric parameters and chemical abundances rather than the recommended GES values. Unfortunately, this node does not analyze GIRAFFE spectra. Although ideally the use of GES GIRAFFE spectra for NGC 4372 would strengthen the conclusion of this paper, the different techniques and methods employed, for this specific science case, would introduce an undesirable heterogeneity.

For our detailed chemical abundance analysis of seven red giant branch (RGB) stars observed with UVES here, the stars were observed in the set-up with central wavelength $580 \mathrm{~nm}$. The spectra were taken in two arms, resulting in a wavelength coverage of 470-684 nm with a gap of $\sim 5 \mathrm{~nm}$ in the center. The FLAMES-UVES fibers have an aperture on the sky of $1^{\prime \prime}$, resulting in a resolving power of $R=47000$. The observations were taken between March 11 and March 16 of 2012 with a mean signal-to-noise ratio in the spectrum of $\sim 50$. Targets for UVES were high-probability cluster members and, in particular, evolved stars. Figure 1 shows the spatial distribution of the seven

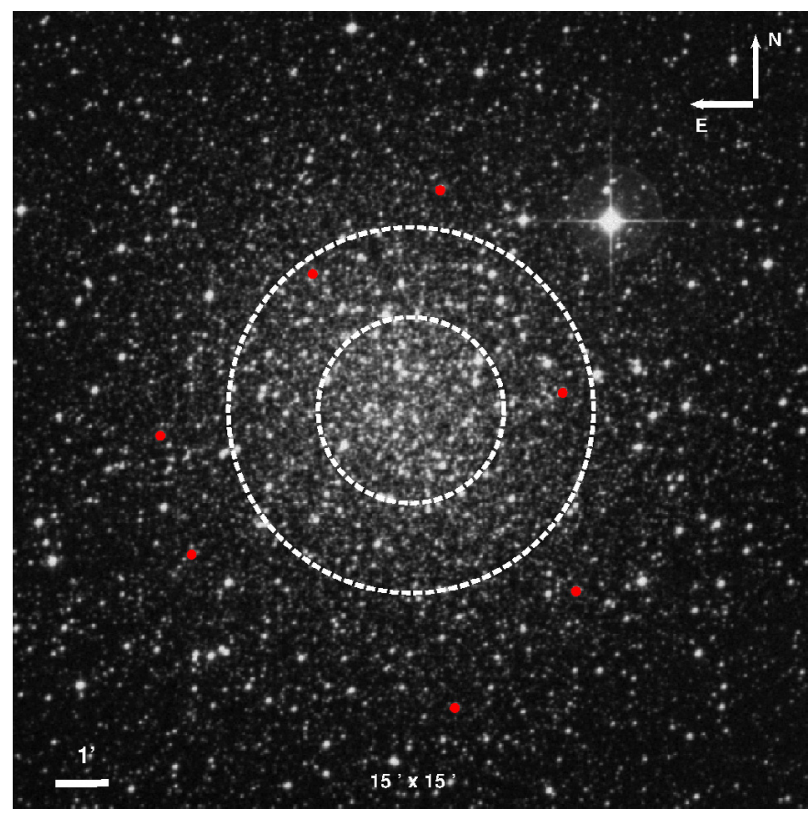

Fig. 1. A $15^{\prime} \times 15^{\prime}$ digitized sky survey image centered on NGC 4372 . North is up and east to the left. The red symbols correspond to the spatial distribution of the 7 stars analyzed. The dashed circles correspond to the core radius, $r_{c}=1.7^{\prime}$ (Harris 1996, 2010 edition), and the half-light radius, $r_{h}=3.4^{\prime}$ (Kacharov et al. 2014).

RGB stars covering a wide area around NGC 4372, while Fig. 2 presents CMDs of the cluster with the analyzed stars indicated. Targets for globular clusters were selected by WG5 (Calibrators and Standards; coordinator: Pancino). More details can be found in Pancino \& Gaia-ESO Survey consortium (2012).

The radial velocity information, available for all the targets observed, is determined within WG8 by cross-correlation against real and synthetic templates. The typical error on the radial velocities of UVES targets is about $0.4 \mathrm{~km} \mathrm{~s}^{-1}$. The mean radial velocity value for the seven targets is $\left\langle v_{\text {rad }}\right\rangle=72.6 \pm$ $1.3 \mathrm{~km} \mathrm{~s}^{-1}$, while the dispersion is $3.6 \mathrm{~km} \mathrm{~s}^{-1}$. The mean radial velocity is in excellent agreement with the value found by Geisler et al. (1995) of $\left\langle v_{\mathrm{rad}}\right\rangle=73.2 \pm 1.4 \mathrm{~km} \mathrm{~s}^{-1}$ where the error is given by the standard error of the mean. More recently, Kacharov et al. (2014) present an extensive kinematic study of this cluster using FLAMES/GIRAFFE observations of 131 stars. They find a mean radial velocity of $v_{\mathrm{r}}=75.9 \pm 0.3 \mathrm{~km} \mathrm{~s}^{-1}$ and a central velocity dispersion of $\sigma_{0}=4.5 \pm 0.3 \mathrm{~km} \mathrm{~s}^{-1}$. As part of the Gaia-ESO survey, the kinematics of seven GGCs is present in Lardo et al. (2015). Using FLAMES/GIRAFFE they analyzed spectra of more than 100 stars and obtained a mean radial velocity of $v_{\mathrm{r}}=75.2 \pm 0.4 \mathrm{~km} \mathrm{~s}^{-1}$ with a velocity dispersion of $3.9 \mathrm{~km} \mathrm{~s}^{-1}$, confirming the membership of our objects. The stellar parameters of stars analyzed in our study are summarized in Table 1. Photometric magnitudes of the observed stars are compiled by GES and presented in Table 2.

\section{Atmospheric parameters and abundance analysis}

The astrophysical parameters obtained from the analysis of the gesiDR2iDR3 data set will be part of the first Gaia-ESO public release of advanced data products. Within WG11, the spectroscopic analysis of UVES data is performed by 13 subgroups, socalled nodes. A multiple parallel analysis of the full data set has 
Table 1. Basic parameters of the observed stars.

\begin{tabular}{cccccccc}
\hline \hline Cname $^{a}$ & $\begin{array}{c}v_{\text {rad }} \\
\left(\mathrm{km} \mathrm{s}^{-1}\right)\end{array}$ & $S N R^{b}$ & $\begin{array}{c}T_{\text {eff }} \\
(\mathrm{K})\end{array}$ & $\begin{array}{c}\log (g) \\
(\mathrm{dex})\end{array}$ & $\begin{array}{c}\xi \\
\left(\mathrm{km} \mathrm{s}^{-1}\right)\end{array}$ & $\begin{array}{c}{[\mathrm{Fe} / \mathrm{H}]} \\
(\mathrm{dex})\end{array}$ \\
\hline 1 & $12250638-7243067$ & $68.6 \pm 0.5$ & 51.17 & $4586 \pm 86$ & $0.70 \pm 0.20$ & $1.56 \pm 0.10$ & $-2.23 \pm 0.21$ \\
2 & $12250660-7239224$ & $67.9 \pm 0.3$ & 34.63 & $4646 \pm 56$ & $1.17 \pm 0.15$ & $1.68 \pm 0.03$ & $-2.22 \pm 0.15$ \\
3 & $12253419-7235252$ & $72.7 \pm 0.7$ & 55.73 & $4527 \pm 33$ & $0.55 \pm 0.07$ & $1.50 \pm 0.17$ & $-2.15 \pm 0.07$ \\
4 & $12253882-7245095$ & $76.0 \pm 0.6$ & 45.45 & $4656 \pm 114$ & $1.19 \pm 0.47$ & $1.56 \pm 0.20$ & $-2.21 \pm 0.20$ \\
5 & $12260765-7236514$ & $75.2 \pm 0.5$ & 71.14 & $4466 \pm 38$ & $0.55 \pm 0.09$ & $1.65 \pm 0.05$ & $-2.20 \pm 0.17$ \\
6 & $12264293-7241576$ & $71.0 \pm 0.7$ & 44.35 & $4712 \pm 87$ & $1.19 \pm 0.21$ & $1.55 \pm 0.13$ & $-2.15 \pm 0.12$ \\
7 & $12264875-7239413$ & $76.9 \pm 0.4$ & 43.89 & $4633 \pm 68$ & $0.84 \pm 0.34$ & $1.54 \pm 0.05$ & $-2.44 \pm 0.16$ \\
\hline
\end{tabular}

Notes. ${ }^{(a)}$ GES object name. It is formed from the coordinates of the object splicing the RA in hours, minutes, and seconds (to 2 decimal places) and the Dec in degrees, minutes, and seconds (to 1 decimal place) together, including a sign for the declination. ${ }^{(b)}$ Mean signal-to-noise ratio in the spectrum.

Table 2. Photometric properties of the observed stars.

\begin{tabular}{ccccccccccc}
\hline \hline Cname $^{a}$ & $\begin{array}{c}B^{b} \\
(\mathrm{mag})\end{array}$ & $\begin{array}{c}V^{b} \\
(\mathrm{mag})\end{array}$ & $\begin{array}{c}I^{b} \\
(\mathrm{mag})\end{array}$ & $\begin{array}{c}J^{c} \\
(\mathrm{mag})\end{array}$ & $\begin{array}{c}H^{c} \\
(\mathrm{mag})\end{array}$ & $\begin{array}{c}K^{c} \\
(\mathrm{mag})\end{array}$ & $\begin{array}{c}E(B-V)^{d} \\
(\mathrm{mag})\end{array}$ & $\begin{array}{c}\mu_{\mathrm{RA}}{ }^{e} \\
(\mathrm{mas} / \mathrm{yr})\end{array}$ & $\begin{array}{c}\mu_{\text {Dec }}{ }^{e} \\
(\mathrm{mas} / \mathrm{yr})\end{array}$ \\
\hline 1 & $12250638-7243067$ & 14.70 & 13.22 & 11.52 & 10.30 & 9.61 & 9.44 & 0.57 & -6.10 & 4.80 \\
2 & $12250660-7239224$ & 15.22 & 13.80 & 12.11 & 10.86 & 10.15 & 9.98 & 0.57 & -12.40 & 6.70 \\
3 & $12253419-7235252$ & 14.83 & 13.21 & 11.29 & 9.92 & 9.18 & 8.97 & 0.59 & -7.10 & 8.60 \\
4 & $12253882-7245095$ & 15.15 & 13.84 & 12.21 & 11.10 & 10.43 & 10.28 & 0.54 & -5.50 & 9.90 \\
5 & $12260765-7236514$ & 14.66 & 13.12 & 11.25 & 9.92 & 9.19 & 8.97 & 0.58 & -13.80 & 9.00 \\
6 & $12264293-7241576$ & 15.08 & 13.76 & 12.14 & 10.98 & 10.30 & 10.16 & 0.54 & -1.10 & 0.60 \\
7 & $12264875-7239413$ & 15.16 & 13.82 & 12.08 & 10.87 & 10.17 & 9.98 & 0.55 & -0.10 & 13.40 \\
\hline
\end{tabular}

Notes. ${ }^{(a)}$ GES object name. It is formed from the coordinates of the object splicing the RA in hours, minutes, and seconds (to 2 decimal places) and the Dec in degrees, minutes, and seconds (to 1 decimal place) together, including a sign for the declination. ${ }^{(b)}$ Magnitudes from APASS (AAVSO photometric All-sky Survey). ${ }^{(c)}$ Magnitudes from 2MASS (Skrutskie et al. 2006). ${ }^{(d)}$ Reddening values from the Galactic dust extinction (Schlegel et al. 1998). ${ }^{(e)}$ Proper motion in RA and Dec from UCAC (Zacharias et al. 2010).

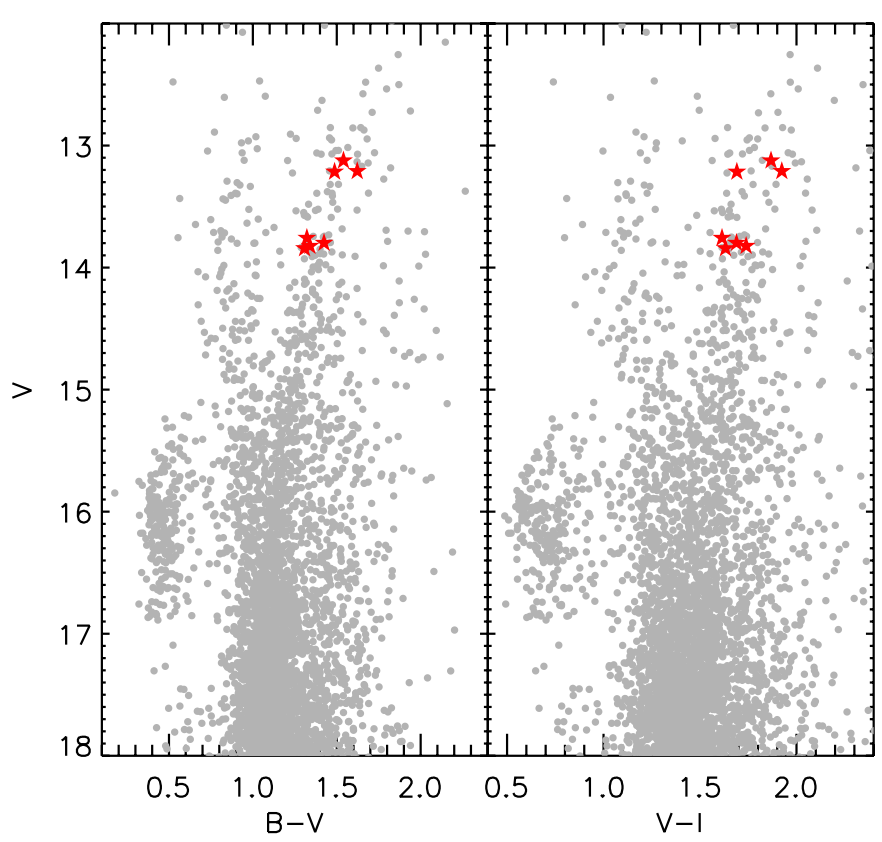

Fig. 2. Color-magnitude diagrams of the globular cluster NGC 4372 on an area of $6.0^{\prime} \times 5.2^{\prime}$. The red symbols correspond to the analyzed RGB stars ${ }^{1}$.

been implemented where different nodes use different methodologies. Unfortunately, only a small number of nodes reported

\footnotetext{
1 The photometric catalog was generated by WG5 based on UBVI archival images and collected at the Wide-Field Imager (WFI) at
}

atmospheric parameters for NGC 4372 stars. As explained in Smiljanic et al. (2014), GES recommended parameters were computed only if at least three nodes provided parameters for a given star. This decision was made based on internal policy rather than the reliability of the values. We decided to make use of Concepcion Node atmospheric parameters and chemical abundances during the following analysis. A critical evaluation of the performance of Concepcion Node, based on a series of calibrators, is presented in Smiljanic et al. (2014). In addition, Concepcion Node is the only one that provides key elements such as $\mathrm{O}$ or $\mathrm{Na}$ for the seven stars observed in NGC 4372.

The atmospheric parameters for NGC 4372 reported by the Concepcion Node correspond to the GES iDR1 (internal data release 1) and were determined by at least five different nodes in a system of multiple parallel analysis. The methodology and codes used by each node are described in detail in Appendix A of Smiljanic et al. (2014), and they range from the classical method of equivalent width (EW) to the use of libraries of observed and/or synthetic spectra. This strategy has two main advantages: it 1) ensures that all sources of errors are well-understood and quantified, including method-dependent effects; 2) ensures that all types of objects can be properly analyzed. To guarantee the homogeneity of the final results, a number of constraints have

the $2.2 \mathrm{~m}$ ESO-MPI telescope (Programs: 164.O-0561 (PI: Krautter), 68.D-0265 (PI: Ortolani), and 69.D-0582 (PI: Ortolani)). The images were prereduced using IRAF package MSCRED (Valdes 1998), while the stellar photometry was derived by using the DAOPHOT II and ALLSTAR programs (Stetson 1987, 1992). Stetson standard fields (Stetson 2000) were used to photometrically calibrate the data, while astrometric calibration was performed with the catalogs UCAC3 (Zacharias et al. 2010) and GSC2 (McLean et al. 2000). 
Table 3. Abundance ratios.

\begin{tabular}{lcrrrrrrrrc}
\hline \hline El. & Method $^{a}$ & Nlines $^{b}$ & 1 & 2 & 3 & 4 & 5 & 6 & 7 & Mean $^{c}$ \\
\hline$[\mathrm{O} / \mathrm{Fe}]$ & SYNTH & 1 & 0.22 & 0.36 & 0.53 & 0.46 & 0.67 & 0.39 & 0.80 & $0.44 \pm 0.07$ \\
{$[\mathrm{Na} / \mathrm{Fe}]_{\text {NLTE }}$} & SYNTH & 2 & 0.39 & 0.91 & 0.53 & 0.69 & 0.07 & 0.67 & 0.53 & $0.54 \pm 0.13$ \\
{$[\mathrm{Mg} / \mathrm{Fe}]$} & EW & 4 & 0.41 & 0.69 & 0.37 & 0.45 & 0.20 & 0.53 & 0.62 & $0.44 \pm 0.07$ \\
{$[\mathrm{Al} / \mathrm{Fe}]$} & EW & 2 & 0.65 & 1.16 & 0.85 & 1.12 & $\ldots$ & 1.01 & 0.01 & $0.96 \pm 0.10$ \\
{$[\mathrm{Si} / \mathrm{Fe}]$} & EW & 1 & $\ldots$ & $\ldots$ & 0.46 & 0.52 & 0.48 & $\ldots$ & 0.77 & $0.48 \pm 0.02$ \\
{$[\mathrm{Ca} / \mathrm{Fe}]$} & EW & 18 & 0.25 & 0.29 & 0.19 & 0.24 & 0.22 & 0.28 & 0.60 & $0.24 \pm 0.02$ \\
{$[\mathrm{Sc} / \mathrm{Fe}]$} & SYNTH & 1 & -0.04 & -0.09 & -0.14 & -0.14 & -0.03 & -0.11 & -0.01 & $-0.09 \pm 0.02$ \\
{$[\mathrm{Ti} / \mathrm{Fe}]^{d}$} & EW & 14 & 0.29 & 0.40 & 0.24 & 0.30 & 0.23 & 0.38 & 0.53 & $0.31 \pm 0.03$ \\
{$[\mathrm{Fe} / \mathrm{H}]$} & EW & 100 & -2.23 & -2.22 & -2.15 & -2.21 & -2.20 & -2.15 & -2.44 & $-2.19 \pm 0.02$ \\
{$[\mathrm{Cr} / \mathrm{Fe}]$} & EW & 6 & -0.38 & -0.20 & -0.33 & -0.37 & -0.36 & -0.28 & -0.15 & $-0.32 \pm 0.03$ \\
{$[\mathrm{Ni} / \mathrm{Fe}]$} & EW & 6 & 0.07 & -0.03 & 0.14 & -0.03 & -0.06 & 0.07 & 0.06 & $0.03 \pm 0.03$ \\
{$[\mathrm{Y} / \mathrm{Fe}]$} & SYNTH & 1 & 0.05 & 0.01 & 0.03 & 0.00 & 0.10 & 0.09 & 0.06 & $0.05 \pm 0.02$ \\
{$[\mathrm{Ba} / \mathrm{Fe}]$} & SYNTH & 1 & -0.29 & -0.15 & -0.09 & -0.16 & -0.11 & -0.17 & -0.23 & $-0.16 \pm 0.03$ \\
{$[\mathrm{La} / \mathrm{Fe}]$} & SYNTH & 1 & 0.09 & -0.03 & -0.36 & -0.31 & -0.06 & -0.06 & 0.14 & $-0.12 \pm 0.08$ \\
\hline
\end{tabular}

Notes. ${ }^{(a)}$ Method used in the abundance determination: SYNTH (spectrum-synthesis method), EW (equivalent width method). ${ }^{(b)}$ Average number of lines employed in the abundance determination. ${ }^{(c)}$ Values obtained excluding Star \#7. ${ }^{(d)}$ Values obtained from the average of Ti I and Ti II.

been imposed. These constraints include the use of a common line list (Heiter et al., in prep.), the use of one single set of model atmospheres (the MARCS models, Gustafsson et al. 2008), the use of a single synthetic spectrum library (de Laverny et al. 2012; Recio-Blanco et al., in prep.), a common solar zero point (Grevesse et al. 2007), and the analysis of common calibration targets. To understand the precision and accuracy of the atmospheric parameters, GES makes use of the Gaia benchmark stars and a set of calibration clusters. The accuracy is judged by the ability of a given node to recover the reference atmospheric parameters of the benchmark stars from their analysis. The precision is judged by the ability of a node to reproduce its own results from multiple analysis of the same star. A detail explanation of the performance of each node, as well as of how the atmospheric parameters were determined, can be found in Smiljanic et al. (2014). Table 1 summarizes the derived atmospheric parameters for NGC 4372 stars. The error reported for each parameter corresponds to the dispersion among the results from different methodologies. This method-to-method dispersion is defined as the degree to which multiple methodologies can agree on the abundance of a star. Although this dispersion is not properly the physical uncertainty of the values, it is a good indicator of the precision and is adopted as the typical uncertainty. To further investigate the reliability of these values, Fig. 3 shows the derived atmospheric parameters of the stars compared with those derived from isochrones.

The chemical abundances for $\mathrm{Mg}, \mathrm{Al}, \mathrm{Si}, \mathrm{Ca}, \mathrm{Ti}, \mathrm{Cr}$, and $\mathrm{Ni}$ were obtained using the EWs of the spectral lines. The EWs were determined with the automatic code DAOSPEC (Stetson \& Pancino 2008). GALA (Mucciarelli et al. 2013) was used to determined the elemental abundances. To complete the analysis and include other key elements, the abundances of $\mathrm{O}, \mathrm{Na}, \mathrm{Sc}, \mathrm{Y}$, $\mathrm{Ba}$, and $\mathrm{La}$ were also obtained. For this set of elements whose lines are affected by blending, the spectrum-synthesis method were used. The local thermodynamic equilibrium (LTE) program MOOG (Sneden 1973) was used for this purpose. Five synthetic spectra having different abundances for each line were calculated, and the best-fitting value estimated as the one that minimizes the RMS scatter. Only lines not contaminated by telluric lines were used. For both methods, the GES guidelines regarding the use of the GES line list (Heiter et al., in prep.), the

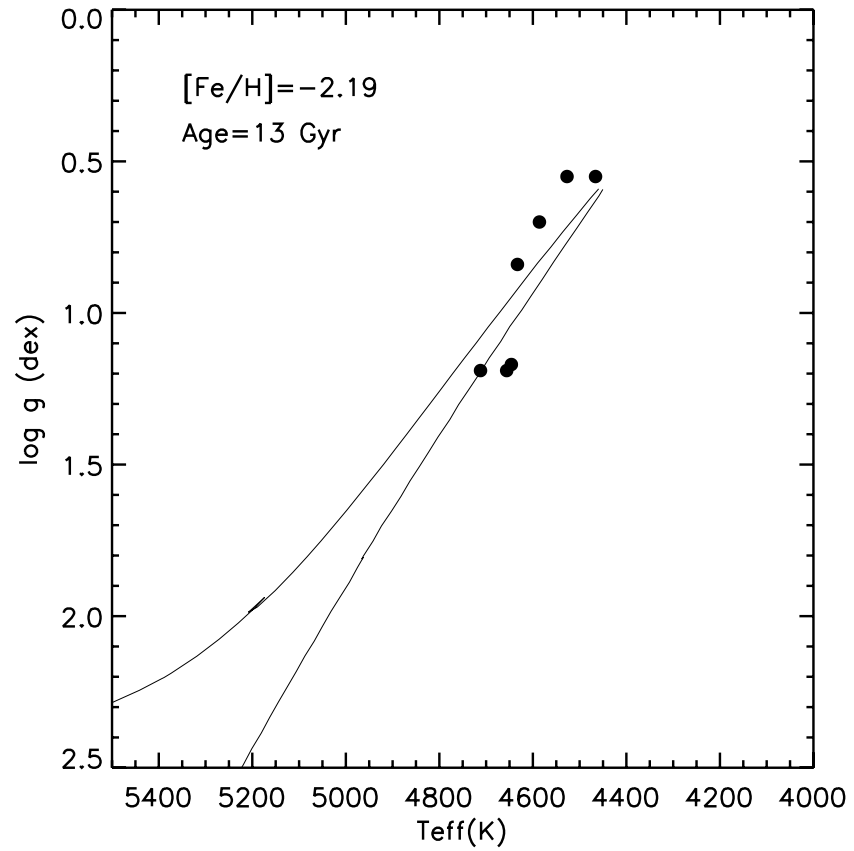

Fig. 3. Parameters of the stars in the $T_{\text {eff }}-\log g$ plane. The solid line corresponds to the isochrone computed with the web tool of the PARSEC group (Bressan et al. 2012).

use of MARCS model atmospheres (Gustafsson et al. 2008) and the use of the Grevesse et al. (2007) solar-zero point were strictly followed. In addition, the $\mathrm{Na}$ abundance was recomputed and a non-LTE correction was applied based on Mashonkina et al. (2000). All these Na abundances were NLTE-corrected. We refer the reader to Smiljanic et al. (2014) for a detailed description of the abundance analysis. The chemical abundances of the observed stars are presented in Table 3.

An internal error (star-to-star) analysis of the derived abundances was performed to properly quantify the internal spread of abundance within a cluster. Table 4 lists the two primary sources of errors contributing to the total budget $\left(\sigma_{\text {tot }}\right)$ : the uncertainties in the measurements and the uncertainties associated 
Table 4. Sensitivity of derived abundances to the atmospheric parameters and EWs.

\begin{tabular}{lcccccc}
\hline \hline & $\Delta T_{\text {eff }}=70(\mathrm{~K})$ & $\Delta \log g=0.19(\mathrm{dex})$ & $\Delta \xi=0.14\left(\mathrm{~km} \mathrm{~s}^{-1}\right)$ & $\sigma_{E W}$ & $\sigma_{\text {tot }}$ & $\sigma_{\text {obs }}$ \\
\hline$\Delta[\mathrm{O} / \mathrm{Fe}]$ & 0.04 & 0.07 & 0.03 & 0.04 & 0.09 & 0.15 \\
$\Delta[\mathrm{Na} / \mathrm{Fe}]$ & 0.00 & -0.07 & -0.03 & 0.04 & 0.09 & 0.29 \\
$\Delta[\mathrm{Mg} / \mathrm{Fe}]$ & 0.11 & -0.08 & -0.07 & 0.02 & 0.15 & 0.16 \\
$\Delta[\mathrm{Al} / \mathrm{Fe}]$ & 0.02 & 0.04 & 0.03 & 0.04 & 0.07 & 0.21 \\
$\Delta[\mathrm{Si} / \mathrm{Fe}]$ & 0.04 & 0.00 & -0.02 & 0.02 & 0.05 & 0.03 \\
$\Delta[\mathrm{Ca} / \mathrm{Fe}]$ & 0.08 & -0.01 & -0.06 & 0.02 & 0.10 & 0.04 \\
$\Delta[\mathrm{Sc} / \mathrm{Fe}]$ & 0.12 & 0.16 & 0.10 & 0.05 & 0.23 & 0.05 \\
$\Delta[\mathrm{Ti} / \mathrm{Fe}]$ & 0.00 & 0.07 & -0.04 & 0.05 & 0.09 & 0.07 \\
$\Delta[\mathrm{Cr} / \mathrm{Fe}]$ & 0.14 & 0.01 & -0.10 & 0.03 & 0.17 & 0.07 \\
$\Delta[\mathrm{Ni} / \mathrm{Fe}]$ & 0.13 & 0.01 & -0.08 & 0.02 & 0.15 & 0.08 \\
$\Delta[\mathrm{Y} / \mathrm{Fe}]$ & 0.03 & 0.07 & -0.01 & 0.06 & 0.10 & 0.04 \\
$\Delta[\mathrm{Ba} / \mathrm{Fe}]$ & -0.09 & -0.06 & -0.02 & 0.06 & 0.13 & 0.07 \\
$\Delta[\mathrm{La} / \mathrm{Fe}]$ & 0.12 & 0.14 & 0.08 & 0.07 & 0.21 & 0.17 \\
\hline
\end{tabular}

Notes. The sensitivity determination was performed for Star \#3. $\sigma_{E W}$ is the error in the measurements. $\sigma_{\text {tot }}$ is the squared root of the sum of the squares of the individual errors. $\sigma_{\mathrm{obs}}$ is the mean observed dispersion.

with the atmospheric parameters. Parameter $\sigma_{\mathrm{EW}}$ corresponds to the mean chemical abundance variation due to error on the EW measurements. Uncertainties in the measurements of EWs were computed by DAOSPEC (Stetson \& Pancino 2008). These confidence intervals estimate the goodness of the fit but also take the quality of the spectrum into account (resolution, signal-tonoise ratio, spectra defects, etc.). These EW errors, $E W_{\text {err }}$, are used by GALA (Mucciarelli et al. 2013) to obtain the uncertainty on the abundance of each element by varying the EW by $1 E W_{\mathrm{err}}$. For the elements whose abundance was obtained by spectrum synthesis, $\sigma_{\mathrm{EW}}$ was obtained as the error given by the fitting procedure. An internal error analysis was performed by varying $T_{\text {eff }}, \log (g)$, and $\xi$ and redetermined abundances of Star \#3, selected as having representative atmospheric parameters. Parameters were varied by $\Delta T_{\text {eff }}=+70 \mathrm{~K}, \Delta \log (g)=+0.19$, and $\Delta \xi=+0.14 \mathrm{~km} \mathrm{~s}^{-1}$, which correspond to the mean error of the sample parameters. We note that these parameter variations are smaller than the standard steps in the MARCS model grid.

The MARCS model interpolator ${ }^{2}$ was used to generate intermediate models from the initial grid. The program interpolates the thermal structure $(T)$, the electronic pressure $\left(P_{\mathrm{e}}\right)$, the gas pressure $\left(P_{\mathrm{g}}\right)$, the opacity $(\kappa)$, and the micro turbulence velocity $(\xi)$ as a function of $T_{\text {eff }}, \log (g)$, and metallicity $[\mathrm{M} / \mathrm{H}]$. The interpolator has been extensively tested on a previous grid of MARCS models that considers the following range of parameters: $3800 \mathrm{~K}<T_{\text {eff }}<7000 \mathrm{~K}, 0.0<\log g<5.0$, and $-4.0<$ $[\mathrm{M} / \mathrm{H}]<0.0$ (Masseron 2006). The interpolation is optimized to account for non-linearities in the grid, and the new interpolated model must lie inside a complete cube of existing models in the parameter space $\left(T_{\text {eff }}, \log g,[\mathrm{M} / \mathrm{H}]\right)$. With the actual grid parameter steps, maximum errors in the interpolated quantities remain below $0.25 \%$ and a few $\%$ for $P_{\mathrm{g}}$ and $P_{\mathrm{e}}$.

Within these limits, we do not consider that the error budget might increase dramatically and that the mean methodto-method dispersion drives the sensitivity to atmospheric parameter variations. This estimation of the internal error was performed following the prescription of Marino et al. (2008). The final total error, $\left(\sigma_{\text {tot }}\right)$, was computed as the square root of the sum of the squares of the individual errors. Table 4 also

\footnotetext{
2 The program and a detailed user manual are available on the MARCS web site: http://marcs.astro.uu.se
}

lists the observed star-to-star dispersion $\left(\sigma_{\mathrm{obs}}\right)$. We would like to point out that our goal is to search for evidence of star-to-star intrinsic abundance variation in each element by comparing the observed dispersion $\left(\sigma_{\text {obs }}\right)$ and the internal errors $\left(\sigma_{\text {tot }}\right)$. For this reason, external sources of error as systematics that do not affect relative abundances are not considered.

\section{Results}

\subsection{Chemical homogeneity of the cluster}

Chemically inhomogeneous populations are observed in virtually all massive, old globular clusters that have been studied to date, but not in open clusters, with the possible exception of NGC 6791 (Geisler et al. 2012, but see Bragaglia et al. 2014 and Cunha et al. 2015). Studying the chemical homogeneity or inhomogeneity of a cluster is necessary to better understand the mechanism of their formation. A large body of evidence now shows that the stars in a GC do not share the same chemical composition (Gratton et al. 2012). As a general rule we can define a globular cluster as an object that is homogeneous in its Fe content and most other heavy elements, but the light elements $\mathrm{Li}$, $\mathrm{C}, \mathrm{N}, \mathrm{O}, \mathrm{Na}, \mathrm{Mg}$, and $\mathrm{Al}$ can show substantial intracluster variations. We investigate the degree of inhomogeneity of the abundances of cluster members in NGC 4372.

Figure 4 shows the abundance ratios versus $[\mathrm{Fe} / \mathrm{H}]$ for individual member stars in the cluster. In each panel, the intersection of the dashed lines delimits a $1 \sigma$ area around the average value. Star-to-star error bar is indicated. Star \#7 stands out as slightly more metal poor than the main body of cluster members. The $\alpha$ elements of this peculiar star also stand out from the cluster mean abundances, except for $\mathrm{Mg}$. The radial velocity and stellar parameters for this object shown in Table 1 agree with those of the rest of the observed stars, but the distinct chemical pattern of this star suggests that it has some kind of anomaly or that it may not be a member. The possibility of Star \#7 being in a binary system would likely alter its radial velocity and artificially lower its derived metallicity owing to the increased continuum flux.

As part of the quality control, GES final products include two binary flags. a) A visual inspection of the cross-correlation function (CCF), computed before co-adding multi-epoch observations, is performed. A star is flagged as a candidate double-lined 


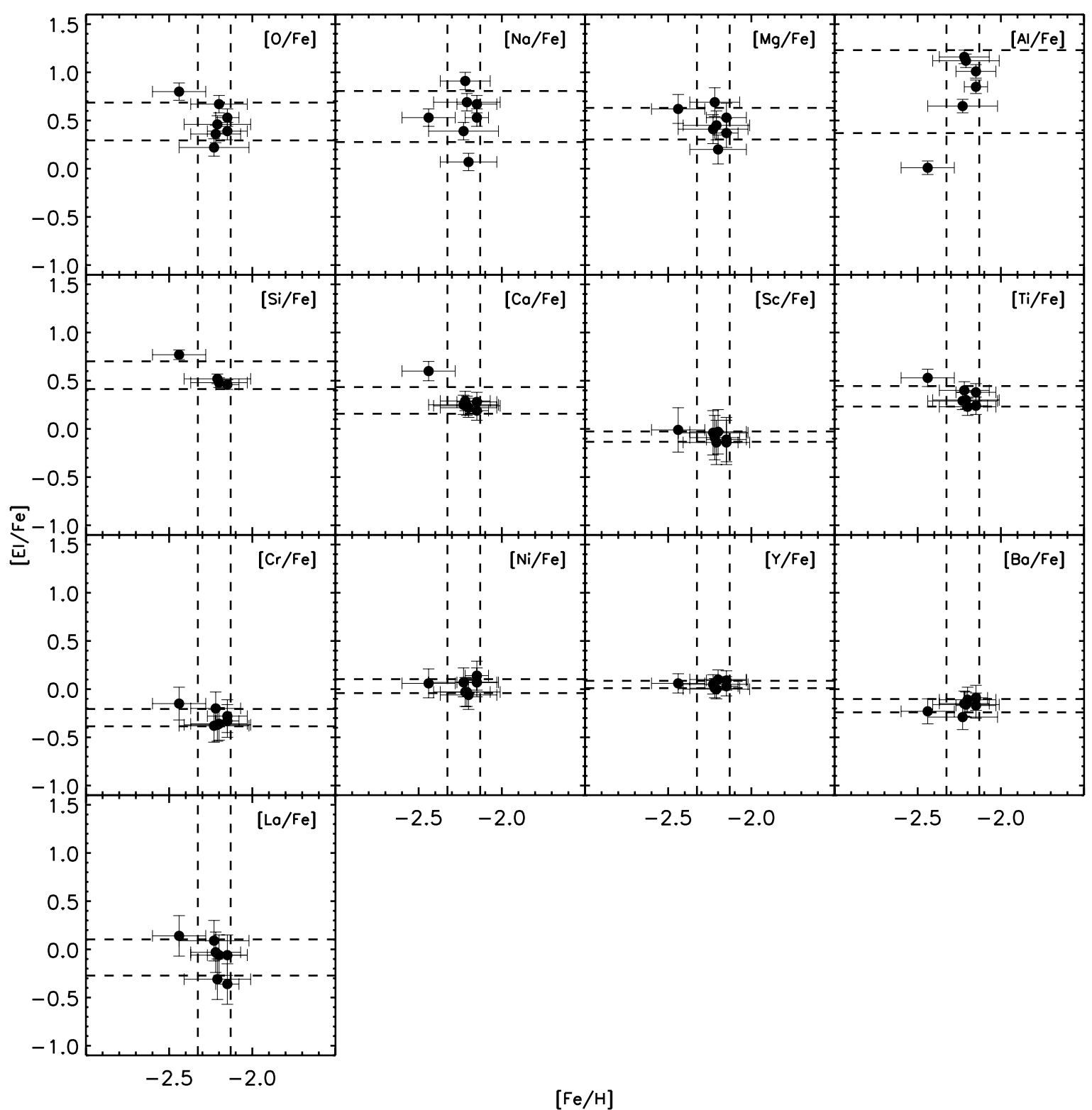

Fig. 4. Abundance ratios versus $[\mathrm{Fe} / \mathrm{H}]$ for individual members of the cluster. The dashed lines indicate the $1 \sigma$ area around the average value.

spectroscopic binary, if the CCFs are characterized by more than one peak or a single peak with strong asymmetries. b) A star is classified as a single-lined spectroscopic binary if the median absolute deviation of multi-epoch repeated measurements of the radial velocity is larger than twice the error on the radial velocity.

None of the NGC 4372 observed stars have been flagged as binary candidates. Given its spatial location, velocity, position in the CMD, and low metallicity, it seems very unlikely that this is a field star. Kacharov et al. (2014) show that according to the prediction of the Besancon Galactic model, only a few field stars with that velocity are expected in the direction of NGC 4372 where none of them are more metal poor than -1.8 dex. This supports the view that Star \#7 is a cluster member. Lapenna et al. (2014) suggest that NLTE effects driven by over ionization mechanisms are present in the atmosphere of AGB stars and significantly affect FeI lines, while leaving FeII features unaltered. This effect could underestimate the metallicity of AGB stars. The low metallicity value of this peculiar star could come from being an AGB star. It is still unclear why the $\alpha$ elements of this star are also significantly different. To be cautious and conservative, we exclude Star \#7 from the analysis.

Figure 5 presents the abundance pattern analyzed in NGC 4372. The box plot illustrates the median and the interquartile range (IQR) of the derived values. Possible outliers are also included where an outlier is defined if it deviates by more than 1.5 IQR. A wide abundance ratio range is present for $\mathrm{O}, \mathrm{Na}$, $\mathrm{Mg}$, and Al. The star-to-star variations are smaller for the heavier elements. La presents a wide abundance range, but we note that the errors in the derived abundances of Sc and La are very large. For both elements, the spectrum-synthesis method with only a single line (Sc II $\lambda 5684.202 \AA$ and La II $\lambda 5122.995 \AA$ ) was used to derived the abundances. The sensitivity of the derived abundances for these two elements to the atmospheric parameters, and specifically to $T_{\text {eff }}$ and $\log g$ (see Table 4 ), is very significant, and any conclusion drawn from these two elements should be treated with caution. To investigate the degree of homogeneity of the abundances further, we compare the scatter produced by 


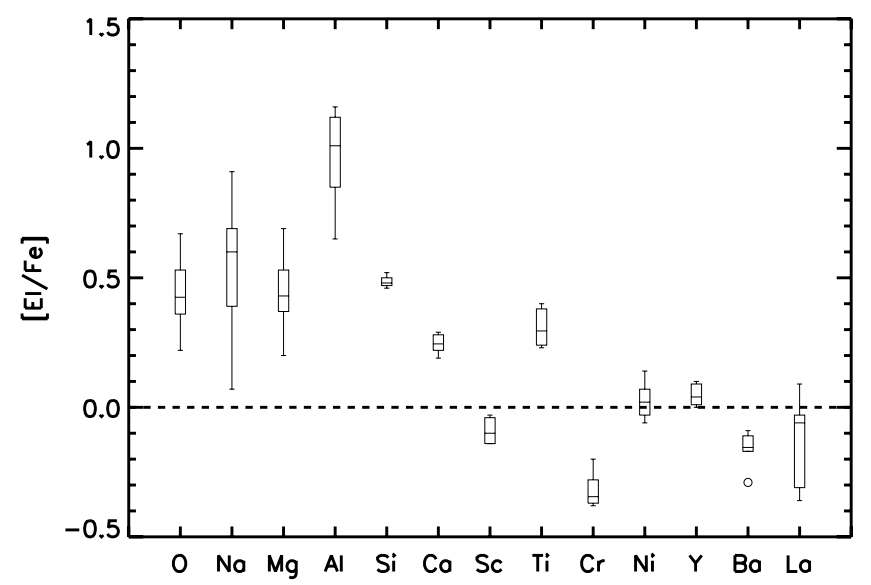

Fig. 5. Box plot of the NGC 4372 star element abundances. Star \#7 has been excluded. For each box, the central horizontal line is the median of the data, while the lower and upper lines indicate the first and third quartiles. The whiskers extend out to the maximum and minimum values of the data. Outliers are identified by small circles.

internal errors, $\left(\sigma_{\text {tot }}\right)$, with the observed dispersion in the chemical abundances, $\left(\sigma_{\text {obs }}\right)$. These values correspond to Cols. 6 and 7 of Table 4. One can consider inhomogeneity when the intrinsic scatter is significantly higher than the expected dispersion given by the internal errors. If we exclude Star \#7 from the error estimation, a clear intrinsic spread can only be identified in $\mathrm{O}, \mathrm{Na}$, and $\mathrm{Al}$.

\subsection{Iron and iron-peak elements}

We obtain a mean metallicity for NGC 4372 of $[\mathrm{Fe} / \mathrm{H}]=-2.23 \pm$ 0.10 . Figure 4 shows no evidence of any intrinsic $\mathrm{Fe}$ abundance spread, with the exception of Star \#7. As discussed in the previous section, this star is anomalous in its chemical behavior, and we prefer to exclude it from cluster means. The issue of intrinsic metallicity spreads in GGCs is of great current interest. Such spreads are found generally only in the most luminous GGCs, with $M_{V} \leq-8.5$. NGC 4372 has $M_{V}=-7.8$, so it is unlikely to host an intrinsic Fe abundance spread. However, we do note that Geisler et al. (1995) did find one of their sample of 11 stars to be significantly (about $0.5 \mathrm{dex}$ ) more metal poor than the rest. This star, like Star \#7, also had a velocity compatible with membership. Excluding Star \#7, we found a mean $[\mathrm{Fe} / \mathrm{H}]$ value of

$[\mathrm{Fe} / \mathrm{H}]=-2.19 \pm 0.03$.

The first attempt to derive a metallicity for NGC 4372 was by Zinn \& West (1984, ZW84), who obtained a value of $[\mathrm{Fe} / \mathrm{H}]=$ $-2.08 \pm 0.15$. Most recently, Geisler et al. (1995) have analyzed the medium-resolution spectra of ten giant stars and obtained, through the near-infrared $\mathrm{Ca}$ II triplet technique, a mean metallicity of $[\mathrm{Fe} / \mathrm{H}]=-2.10 \pm 0.04$. Several other authors have attempted to derive the metallicity based on similar techniques but using different scales with a variety of results (e.g., $[\mathrm{Fe} / \mathrm{H}]_{Z W 84}=-2.03$; Rutledge et al. 1997). Carretta et al. (2009c) adopt a new scale that is a fundamental shift from the older and widely used ZW84 metallicity scale. The authors argue that this traditional scale was calibrated against only a handful of high-dispersion spectroscopic $[\mathrm{Fe} / \mathrm{H}]$ values available at that time. Carretta et al. (2009c) define an accurate and updated metallicity scale using high-dispersion and high signal-to-noise spectroscopic measures of 19 GCs covering the metallicity range

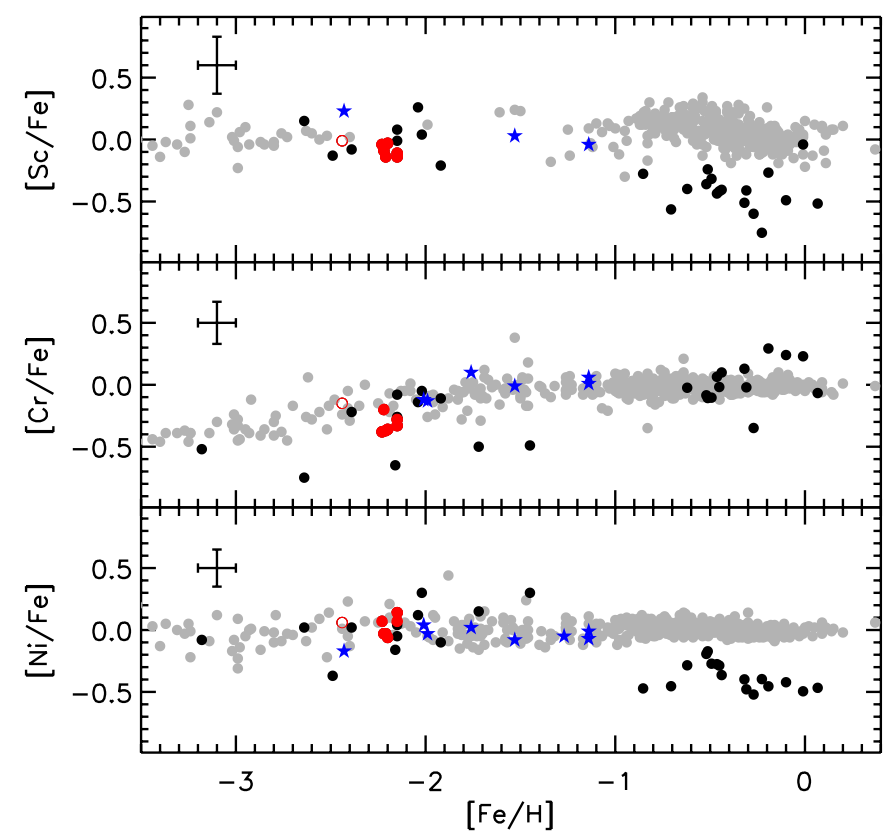

Fig. 6. Iron-peak element ratios ([Sc/Fe], $[\mathrm{Cr} / \mathrm{Fe}],[\mathrm{Ni} / \mathrm{Fe}])$ versus $[\mathrm{Fe} / \mathrm{H}]$. Red symbols correspond to values for NGC 4372 from the present study where the open red circle corresponds to peculiar Star \#7. Blue stars are Galactic globular clusters (Ivans et al. 2001; Lee et al. 2005; Lee \& Carney 2002; Carretta 2006; Carretta et al. 2009a, 2010a; Villanova \& Geisler 2011; Muñoz et al. 2013; Koch \& McWilliam 2011). Gray symbols are halo and disk stars (Fulbright 2000; Reddy et al. 2003, 2006; Cayrel et al. 2004), and black symbols correspond to extragalactic objects: Draco, Sextans, Ursa minor, Carina, and Sagittarius dwarf galaxy (Shetrone et al. 2001; Sbordone et al. 2007; Koch et al. 2008a) and the ultra-faint dwarf spheroidals Boötes I and Hercules (Ishigaki et al. 2014; Koch et al. 2008b). A star-to-star error bar is indicated.

of the bulk of GGCs. Based on this scale, they provide a value of $[\mathrm{Fe} / \mathrm{H}]=-2.19 \pm 0.08$ for NGC 4372 metallicity, using Geisler et al. (1995) Ca II triplet data. This updated result is in excellent agreement with our metallicity. The RMS scatter of our metallicity is a measure of the intrinsic spread of iron in the cluster. Carretta et al. (2009c) confirm that the scatter in Fe of most GCs is very small with an upper limit of less than 0.05 dex. Our observed scatter is consistent with what is expected from errors, so we conclude that there is no clear metallicity spread in NGC 4372, with the caveat that Star \#7 is considered either an outlier for other reasons or a non-member.

The chemical abundances for the iron-peak elements $\mathrm{Sc}, \mathrm{Cr}$, and $\mathrm{Ni}$ are listed in Table 3. The values are solar within the errors except for the $\mathrm{Cr}$ abundance which is underabundant. Figure 6 shows the elemental abundance of each star compared with a variety of Galactic and extragalactic objects. We have included values from GGCs (Ivans et al. 2001; Lee et al. 2005; Lee \& Carney 2002; Carretta 2006; Carretta et al. 2009a, 2010a; Villanova \& Geisler 2011; Muñoz et al. 2013; Koch \& McWilliam 2011), disk and halo stars (Fulbright 2000; Reddy et al. 2003, 2006; Cayrel et al. 2004), and extragalactic objects, such as Draco, Sextans, Ursa Minor, the Sagittarius dwarf galaxy, and the ultrafaint dwarf spheroidals Boötes I and Hercules (Shetrone et al. 2001; Sbordone et al. 2007; Ishigaki et al. 2014; Koch et al. 2008a,b).

In general, we find that NGC 4372 stars have abundances of these elements that agree with those of other GCs and halo field stars of similar metallicity. 


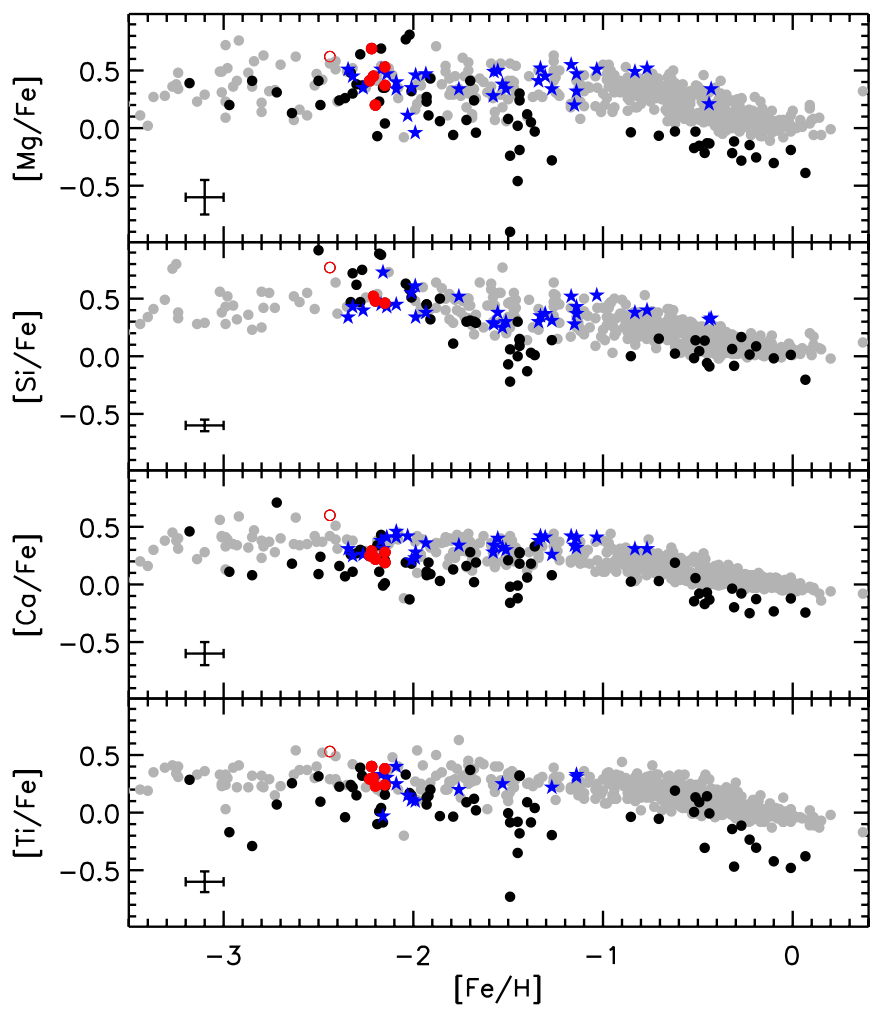

Fig. 7. $\alpha$ element ratios $([\mathrm{Mg} / \mathrm{Fe}],[\mathrm{Si} / \mathrm{Fe}],[\mathrm{Ca} / \mathrm{Fe}],[\mathrm{Ti} / \mathrm{Fe}])$ versus $[\mathrm{Fe} / \mathrm{H}]$. Symbols are as in Fig. 6.

\section{3. $\alpha$ elements}

All the $\alpha$ elements listed in Table $3(\mathrm{Mg}, \mathrm{Si}, \mathrm{Ca}, \mathrm{Ti})^{3}$ are overabundant compared to the Sun. This is a common feature among almost all GCs, as well as among similarly metal-poor halo field stars in the Galaxy. A glaring exception is Rup 106 (Villanova et al. 2013), which shows solar $\alpha$ element abundances. Figure 7 shows the $\alpha$ element $(\mathrm{Mg}, \mathrm{Si}, \mathrm{Ca}, \mathrm{Ti})$ ratios as a function of metallicity. For comparison purposes, we have included values from GGCs, disk, and halo stars and extragalactic objects. The sources of the data are the same as those given in the previous section. The $\alpha$ elements in NGC 4372 seem to follow the same trend as GGCs and are fully compatible with halo field stars. Star \#7 stands out from the cluster behavior, suggesting once again the singularity of this star. Excluding Star \#7, we derive for NGC 4372 a mean $\alpha$ element abundance of

$[\alpha / \mathrm{Fe}]=+0.37 \pm 0.07$

Figure 8 represents the $[\alpha / \mathrm{Fe}]$ versus $[\mathrm{Fe} / \mathrm{H}]$ relation using the symbols and colors defined in Fig. 7. Similar to halo Milky Way stars, GGCs show a constant overabundance of $\alpha$ elements over a wide range of metallicities $([\mathrm{Fe} / \mathrm{H}]<-1)$. In contrast, metalrich dwarf spheroidal galaxies, $[\mathrm{Fe} / \mathrm{H}]>-2$, tend to have a much lower $\alpha$ content than Galactic objects at similar metallicity, in some cases leading to even subsolar ratios (Geisler et al. 2007). NGC 4372 falls in a region where both Galactic and extragalactic objects overlap in their $\alpha$ element content, so it is not possible to draw conclusions regarding its origin from this diagram.

\footnotetext{
3 Since $\mathrm{O}$ shows a star-to-star variation and the $\mathrm{Na}-\mathrm{O}$ anticorrelation (see Sect. 4.4), it will be treated separately.
}

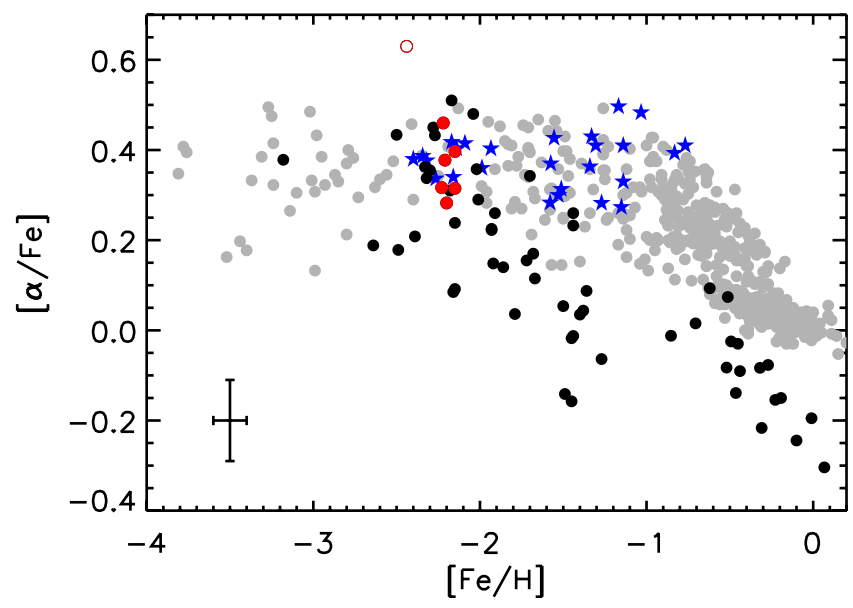

Fig. 8. Mean $\alpha$ element abundance versus $[\mathrm{Fe} / \mathrm{H}]$. Symbols are as in Fig. 6.

\subsection{Na-O anticorrelation}

The Na-O anticorrelation is the classical signature of the process of proton-capture reactions in H-burning at high temperature in a previous generation of stars. All those GGCs studied in detail to date show this feature with the possible exception of Ruprecht 106 (Villanova et al. 2013). It is important to mention that such abundance trends are not seen in the halo field stars with similar metallicity. As described in the Introduction, this chemical signature has been proposed for defining a GC (Carretta et al. 2010c). Figure 9 shows the Na-O abundances in the stars of our sample. For comparison purposes, abundances of GGCs and halo and disk field stars have been overplotted. In addition, two distinct $\mathrm{Na}-\mathrm{O}$ anticorrelations have been plotted with lines that correspond to the dilution models determined by Carretta et al. (2009b). One is O richer and is represented by the trend of the stars in NGC $7078(\mathrm{M} 15)([\mathrm{Fe} / \mathrm{H}]=-2.31)$, while the other is $\mathrm{O}$ poorer and is represented by the trend of the stars in NGC $2808([\mathrm{Fe} / \mathrm{H}]=-1.51)$. Following Carretta et al. (2009b), we have obtained the dilution model for NGC 4372. NGC 4372 stars have a clear intrinsic dispersion and apparent anticorrelation, which corroborate the chemical inhomogeneities found in Sect. 4.1. Figure 9 confirms the initial assumption that NGC 4372 is a multiple-population GC. Star \#1 shows a value of $[\mathrm{O} / \mathrm{Fe}]$ that is significantly lower than in the rest of the sample. This star does not follow the Na-O anticorrelation described by the rest of the stars in NGC 4372. The $\mathrm{O}$ abundances were obtained using the line $\lambda 6300.30 \AA$. This line is significantly weaker in Star \#1 than in the rest of the sample. In addition, NGC 4372 stars inhabit an area in the figure that follows the general GCs trend, although our sample lies at the high $[\mathrm{O} / \mathrm{Fe}]$ end.

Some interesting characteristics can be identified among our sample. One star is very Na-poor/O-rich, which would correspond to the putative primordial stellar component, while a group of Na-rich/O-poor stars would be associated with a second generation of stars. However, the low number statistics doesn't let us confirm that split. Our sample does not seem to show any star with very large O-depletion that would lie in the extreme region, resembling the behavior of other metal-poor clusters, such as NGC 7078 (M15), NGC 7099, or NGC 4590 (Carretta et al. 2009b).

The dilution model considered by Carretta et al. (2009b) to explain the $\mathrm{Na}-\mathrm{O}$ anticorrelation (Fig. 9) makes the basic 


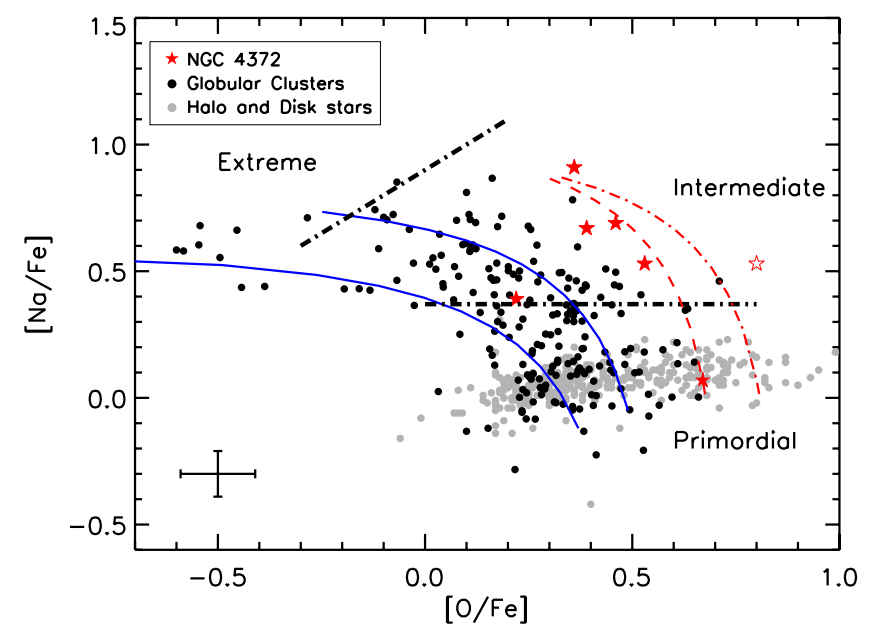

Fig. 9. $[\mathrm{O} / \mathrm{Fe}]$ vs. $[\mathrm{Na} / \mathrm{Fe}]$. Red symbols correspond to this study where the open red symbol corresponds to the peculiar Star \#7. Black symbols correspond to GGC data from Carretta et al. (2009a), and gray symbols to halo and disk field stars from Fulbright (2000), Reddy et al. (2003), and Reddy et al. (2006). Blue solid lines correspond to the dilution model from Carretta et al. (2009b) for NGC 7078 (M15) and NGC 2808. The red dash-dotted line is the dilution model for NGC 4372 considering the full sample of stars and the red dashed line is the dilution model but excluding Star \#7. The black lines are the empirical separations into primordial, intermediate, and extreme populations according to Carretta et al. (2009b). The star-to-star error bar is indicated.

assumptions: a) the polluting material has a well-defined composition and $b$ ) that polluting material is then diluted with a variable amount of primordial material producing the characteristic pattern of the Na-O anticorrelation. The minimum $\mathrm{Na}$ and maximum $\mathrm{O}$ abundances in each cluster represent the original $\mathrm{Na}$ and $\mathrm{O}$ composition of the cluster. NGC 4372 has abundances for $\mathrm{O}$ that are slightly high compared with the sample of Carretta et al. (2009b). High values of O content (as an $\alpha$ element) would imply a marginal contribution by Type-Ia SNe to their original composition. However, the rest of the $\alpha$ abundances ( $\mathrm{Mg}, \mathrm{Si}, \mathrm{Ca}$, and Ti) analyzed in NGC 4372 are fully compatible with GGCs (Figs. 7 and 8). The apparent offset of the Na-O anticorrelation observed in Fig. 9 could indicate that NGC 4372 was formed in an environment with high $\mathrm{O}$ for its metallicity.

\section{5. $M g-A l$ cycle}

In addition to the $\mathrm{CNO}$ and $\mathrm{Ne}-\mathrm{Na}$ cycles, there is evidence that the $\mathrm{Mg}$-Al cycle is also active in GC polluters. Models predict that Al should show correlations with elements that are enhanced by the action of the $\mathrm{Ne}-\mathrm{Na}$ (such as $\mathrm{Na}$ ) and $\mathrm{Mg}-\mathrm{Al}$ cycles and should anti-correlate with elements that are depleted in $\mathrm{H}$-burning at a high temperature (such as $\mathrm{O}$ and $\mathrm{Mg}$ ) (see discussion in Gratton et al. 2012). We analyzed the abundances of some proton-capture elements to identify any (anti)correlation in the cluster.

The pattern of abundances of the elements participating in proton-capture reactions (Na, Al, Mg, Si) observed in NGC 4372 giant stars is summarized in Figs. 10 and 11. The level of scatter in all these plots is significant. For comparison purposes, abundances of GGCs and halo and disk field stars have been overplotted. If the temperature is high enough, one should expect some degree of anticorrelation between $\mathrm{Mg}$ and $\mathrm{Al}$; however, the production of $\mathrm{Al}$ at the expense of $\mathrm{Mg}$ via the $\mathrm{MgAl}$-cycle

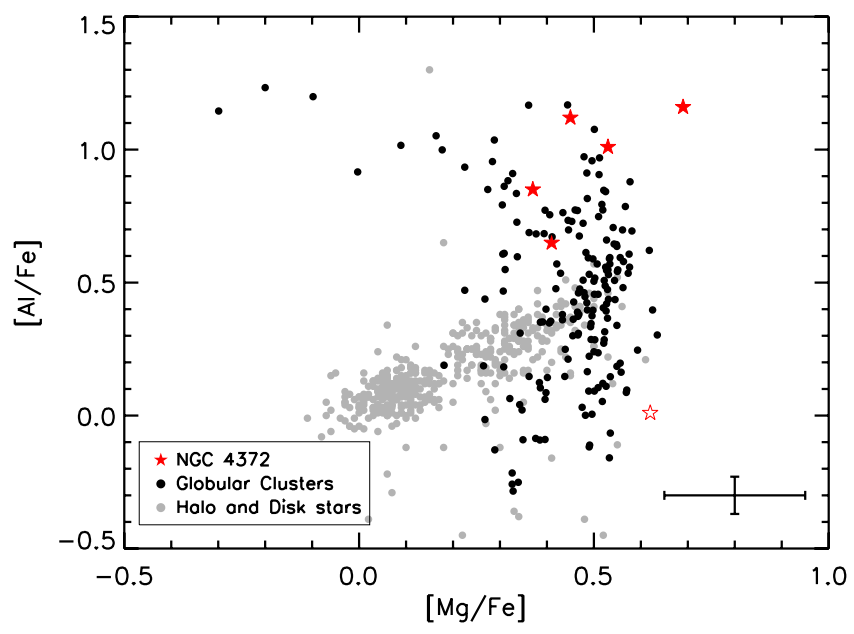

Fig. 10. $[\mathrm{Mg} / \mathrm{Fe}]$ vs. $[\mathrm{Al} / \mathrm{Fe}]$. Symbols are as in Fig. 9. Star-to-star error bar is indicated.

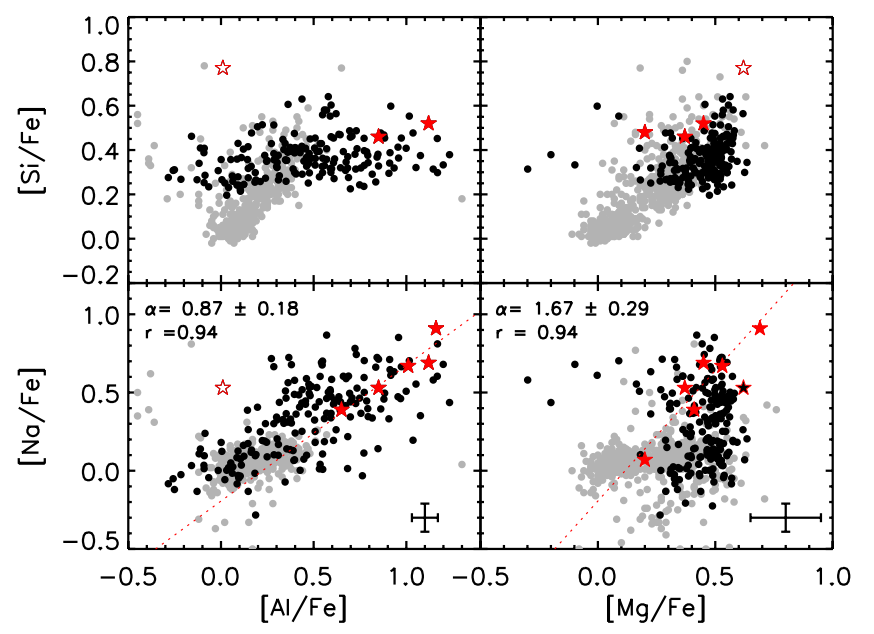

Fig. 11. Same as Fig. 10 but for $[\mathrm{Si} / \mathrm{Fe}]$ versus $[\mathrm{Al} / \mathrm{Fe}]$, $[\mathrm{Si} / \mathrm{Fe}]$ versus $[\mathrm{Mg} / \mathrm{Fe}],[\mathrm{Na} / \mathrm{Fe}]$ versus $[\mathrm{Al} / \mathrm{Fe}]$, and $[\mathrm{Na} / \mathrm{Fe}]$ versus $[\mathrm{Mg} / \mathrm{Fe}]$. Symbols are as in Fig. 9. Star-to-star errors bars are indicated in each panel. Dotted red lines correspond to the linear fit excluding star \#7. The slope of each fit is represented as $\alpha$ with the corresponding sigma error. The parameter $r$ corresponds to the Pearson correlation coefficient.

does not result in such a well-defined anticorrelation as the NaO one (Langer \& Hoffman 1995). In fact, large variations in Al are often accompanied by much smaller changes in $\mathrm{Mg}$ abundances (Carretta et al. 2009a). The Mg-Al behavior in NGC 4372 (Fig.10) presents similar features to the ones found in studies of other GGCs (Carretta et al. 2009a).

A clear $\mathrm{Al}$ spread is present in our sample with a star-tostar variation range in the $\mathrm{Al}$ abundances of $\sim 0.5$ dex (excluding Star \#7), but we do need to consider the potential influence of NLTE effect in Al abundances. Thygesen et al. (2014) show that there is a non-negligible NLTE effect on at least some Al lines. This NLTE effect correlates with $T_{\text {eff }}$ where cooler stars would show stronger negative NLTE correction that warmer ones. Taking the narrow range of stellar parameters (and in particular $T_{\text {eff }}$ ) of our sample stars into account, the Al spread should not be affected significantly by NLTE corrections. On the other hand, the larger uncertainty on $\mathrm{Mg}$ suggests that $\mathrm{Mg}$ may be consistent with no spread. The correlation detected among stars of NGC 4372 follows the behavior 
displayed by GGC stars with a clear distinction from disk and halo field stars. Unlike the well-known O-Na anticorrelation, the $\mathrm{Mg}-\mathrm{Al}$ anticorrelation is more difficult to reproduce in simulations (Denissenkov et al. 1998; Ventura et al. 2001). Carretta et al. (2009a) show that Al-rich and Mg-depleted stars are only present in massive clusters (NGC 2808, NGC 6388, NGC 6441), metal-poor clusters (NGC 6752), or both (NGC $7078=\mathrm{M} 15$ ). In those clusters, a clear $\mathrm{Mg}-\mathrm{Al}$ anticorrelation is observed even among main sequence stars (Bragaglia et al. 2010). More precise $\mathrm{Mg}$ values would be required to clarify the present of a $\mathrm{Mg}$ spread in NGC 4372. The presence of a Mg-Al anticorrelation in NGC 4372 would indicate that the polluted generation has been enriched by material from stars where the $\mathrm{Mg}$-Al burning cycle was active.

The top panels in Fig. 11 show the abundances of elements involved in the $\mathrm{Ne}-\mathrm{Na}, \mathrm{Mg}-\mathrm{Al}$ cycles of proton-capture reactions in high-temperature H-burning. Carretta et al. (2009a) find that stars with extreme Al overabundance also show Si enhancement with respect to the remaining stars in the same cluster. Again, this effect is limited to massive or metal-poor GGCs. The correlation between $\mathrm{Si}$ and $\mathrm{Al}$ abundances is a signature of production of ${ }^{28} \mathrm{Si}$ from the $\mathrm{Mg}-\mathrm{Al}$ cycle (Yong et al. 2005). The reaction producing this isotope becomes predominant in the $\mathrm{Mg}-\mathrm{Al}$ cycle when the temperature is very high (exceeding $T_{6}=65 \mathrm{~K}$ ) (Arnould et al. 1999). The chemical pattern observed in these giant stars must be imprinted by a previous generation of massive stars to be capable of reaching those high temperatures. If we exclude Star \#7 from the analysis, Si does not show large star-to-star variations, but unfortunately the number of stars with reliable $\mathrm{Si}$ abundances are very limited. A clear $\mathrm{Na}-\mathrm{Al}$ correlation is evident in our data. This is a reflection of the relation between the $\mathrm{Mg}-\mathrm{Al}$ and $\mathrm{Ne}-\mathrm{Na}$ cycles (see discussion in Gratton et al. 2012). In fact, $\mathrm{Al}$ and $\mathrm{Na}$ are predicted to be simultaneously enhanced when the $\mathrm{Ne}-\mathrm{Na}$ and $\mathrm{Mg}-\mathrm{Al}$ cycles are both acting. A $\mathrm{Na}-\mathrm{Mg}$ relation is also identified in NGC 4372 stars. This behavior does not follow the general trend of GGCs, and in fact one would expect to find a $\mathrm{Na}-\mathrm{Mg}$ anticorrelation. This relation is driven mostly by only two stars. Large Mg errors could cause this unexpected relation. More extensive samples of stars with accurate determinations of abundances are required for a more detailed analysis.

\subsection{Heavy elements}

While light-element variations are well known in GCs, intrinsic dispersion among heavier elements is less common. Most of the heavier elements $(Z>30)$ are produced by either slow or rapid neutron-capture reactions (the so-called $s$ - and $r$-processes). The s-process happens in a different physical condition with respect to $r$-process and are thus likely to happen in different astrophysical sites. We measured the abundances of the neutron-capture elements: Y, La, and $\mathrm{Ba}$. These elements are mainly produced by the $s$-process at solar metallicity where the neutron-capture time is much longer than the beta-decay lifetime. From our data we could not determine the $r$ - and $s$-process ratio of neutron capture, because we did not measure a reliable abundance for a typical $r$-process species (e.g., Eu).

The abundance pattern of heavy neutron-capture elements (Y, Ba, La) is shown in Fig. 12. We have overplotted the pure $r$ - and $s$-process patterns from Simmerer et al. (2004). The $\mathrm{Ba}$ abundance was used to set the zero point of the curves. Although the analysis includes very few points for any solid conclusion, the observed abundance of $\mathrm{Y}$ can be reproduced by a pure $r$-process pattern.

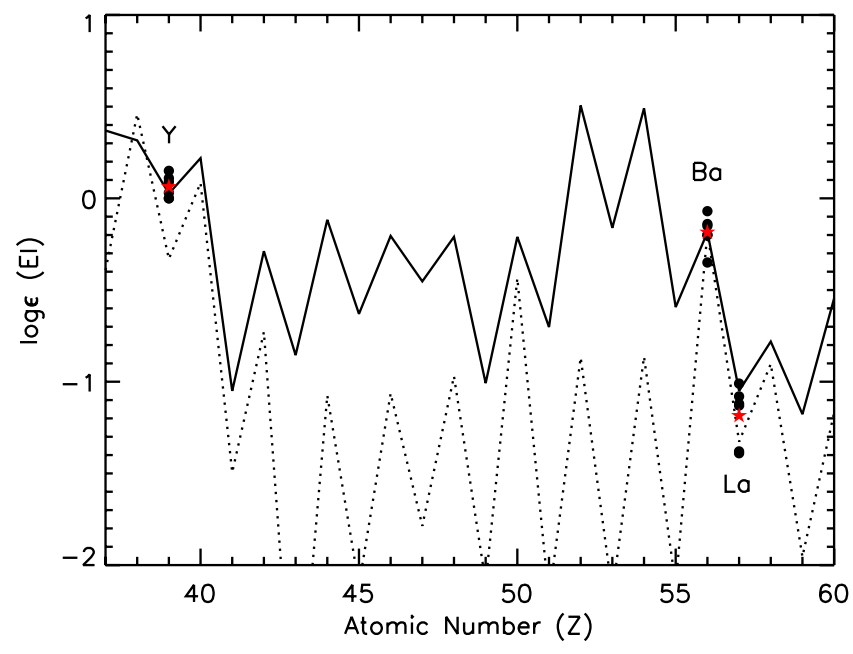

Fig. 12. Abundance distribution of heavy neutron-capture elements in NGC 4372. Black symbols correspond to the individual stars, and red symbols represent the mean abundance for the cluster (excluding Star \#7). Solid and dashed lines show the scaled-solar $r$ - and $s$-process patterns, respectively (Simmerer et al. 2004) and have been normalized to the mean abundance of $\mathrm{Ba}$. Notation: $\log \epsilon(\mathrm{El})=$ $\log _{10}\left(N_{\mathrm{El}} / N_{\mathrm{H}}\right)+12.0$

Figure 13 shows the abundance ratios of the heavy elements analyzed as a function of $[\mathrm{Na} / \mathrm{Fe}]$ and $[\mathrm{Al} / \mathrm{Fe}]$. The $[\mathrm{Ba} / \mathrm{Fe}]$ abundance ratio is subsolar, in agreement with recent studies that found that $[\mathrm{Ba} / \mathrm{Fe}]$ decreases with low metallicites and becomes subsolar at least in field stars with $[\mathrm{Fe} / \mathrm{H}]<-2.0$ (e.g., Gratton $\&$ Sneden 1994; Fulbright 2002). These subsolar values will agree with recent studies that indicate that metal-poor globular clusters primarily exhibit $r$-process signatures, owing to the inefficiency of $s$-process in low-metallicity environments (Gratton et al. 2004; Roederer et al. 2010; Koch et al. 2009). At $[\mathrm{Fe} / \mathrm{H}]<-2$ dex, the environment was not yet polluted by a sufficient number of low-mass AGB stars, and the average $s$-process abundances are low. All the elements show an apparent small dispersion, however the observed range is very comparable to that expected from the errors (see Table 4) so we cannot confirm any clear evidence for an intrinsic spread in any of these elements.

Only a handful of metal-poor globular clusters show a potential star-to-star dispersion in neutron-capture elements (Roederer 2011; Kacharov et al. 2013). Marino et al. (2009) find a wide range of abundances values for $s$-process elements, Y, Zr, and $\mathrm{Ba}$, in M22. They also identified a bimodality among these elements. None of the elements show a correlation with $\mathrm{Na}, \mathrm{O}$, and Al. The bimodality in s-process elements in M22 resembles the case of NGC 1851 (Yong \& Grundahl 2008), although in this case the $s$-element abundance appears to correlate with the $\mathrm{Na}, \mathrm{Al}$, and $\mathrm{O}$ abundances. The metal-poor globular cluster M 15, with $[\mathrm{Fe} / \mathrm{H}]=-2.31 \pm 0.06$ dex (Carretta et al. 2009b), has also proven to be an interesting case for study. Sneden et al. $(1997,2000)$ found a scatter of heavy neutron-capture elements but no significant $s$-process enrichment in M15. More recently, Worley et al. (2013) have found a bimodal distribution of Ba, Eu, and possibly La in M15. Both modes of the bimodality seem to be indicative of a pollution scenario dominated by the $r$-process and only varying because of different degrees of enrichment. A group of La-rich and La-poor stars can be identified in our sample, but such a small sample does not allow us to carry out a further investigation. 

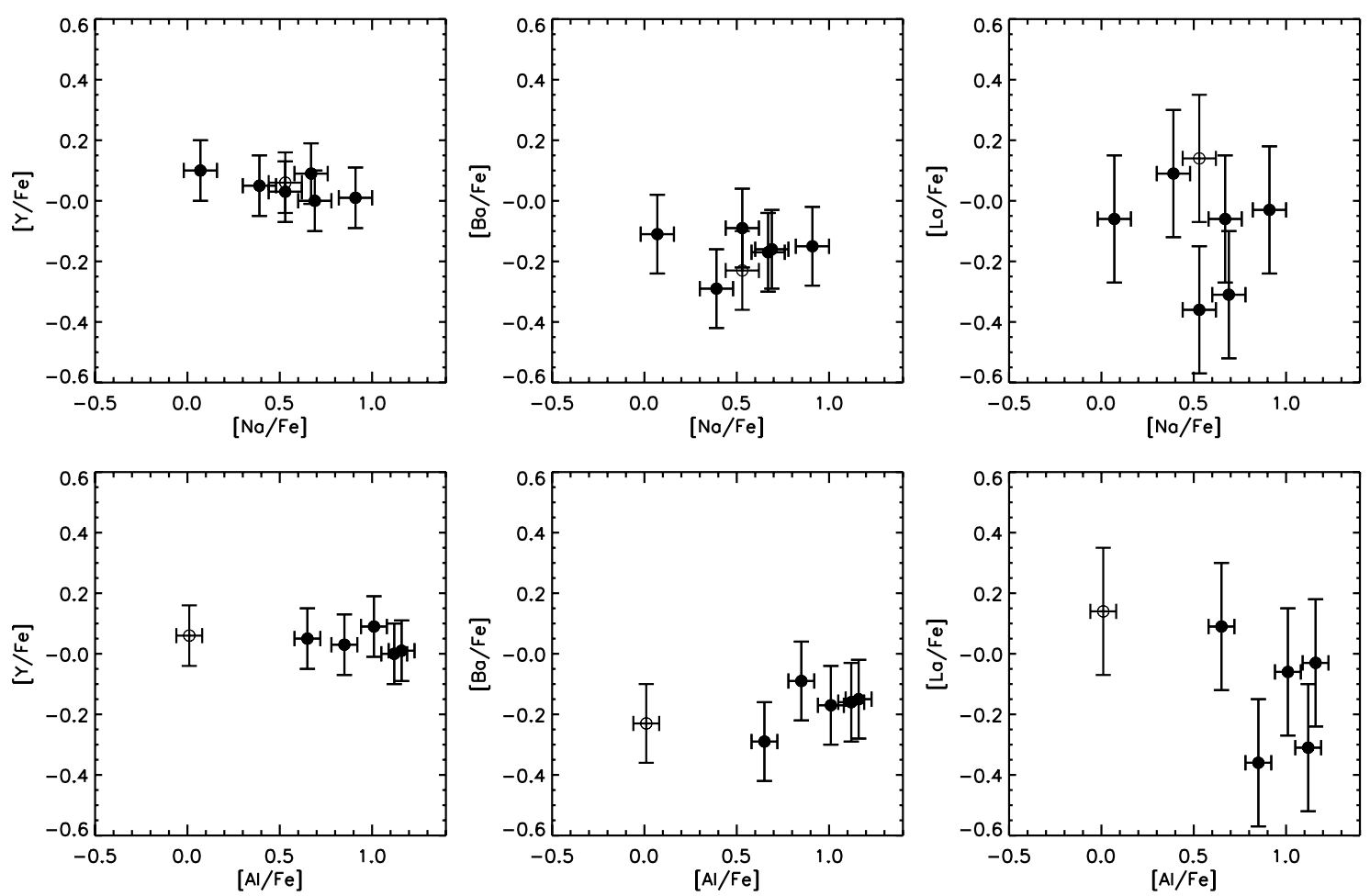

Fig. 13. Neutron capture elements $[\mathrm{Y} / \mathrm{Fe}],[\mathrm{Ba} / \mathrm{Fe}]$, and $[\mathrm{La} / \mathrm{Fe}]$ as a function of light elements $[\mathrm{Na} / \mathrm{Fe}]$ and $[\mathrm{Al} / \mathrm{Fe}]$. The open symbol corresponds to Star \#7.

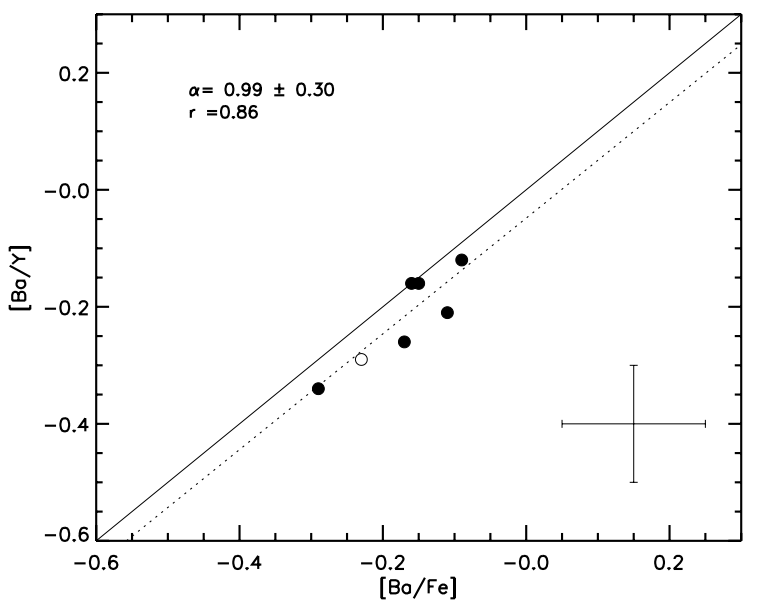

Fig. 14. Relation of the light and heavy $s$-process elements, $[h s / l s]=[\mathrm{Ba} / \mathrm{Y}]$, as a function of $[\mathrm{Ba} / \mathrm{Fe}]$. The open symbol corresponds to Star \#7. The dashed line corresponds to the linear fit excluding Star \#7. The solid line corresponds to the 1:1 correlation. The slope of the fit is represented as $\alpha$ with the correspondent sigma error. The Pearson correlation coefficient is also shown.

The weak $s$-process accounts for the major fraction of the light $s$-process $(l s-)$ elements, like $\mathrm{Y}$, and occurs in core Heburning massive stars $\left(M>8 M_{\odot}\right)$ (Pignatari et al. 2010). The main $s$-process takes place in thermally pulsating AGB stars $\left(1 M_{\odot}<M<8 M_{\odot}\right)$ producing light $s$-process $\left(l s^{-}\right)$and heavy $s$-process $\left(h s^{-}\right)$elements, such as Ba and La (Arlandini et al. 1999). Therefore, analysis of ratios between $l s$ - and $h s$ - elements are very interesting for constraining these formation processes. Figure 14 shows the $[h s / l s]=[\mathrm{Ba} / \mathrm{Y}]$ ratio as a function of the heavy $s$-process element Ba. Although the large errors should be considered, a relation is present between $\mathrm{Y}$ and $\mathrm{Ba}$. This relation could imply a definite spread of Y and Ba. Although the spread among heavy elements is comparable to the star-to-star scatter found in $[\mathrm{Fe} / \mathrm{H}]$, heavy element absorption lines are generally difficult to measure. For example, Ba absorption lines are quite strong among luminous RGB stars, so saturation could be a problem. They have hyperfine structure and involve the choice of atmospheric parameters. In particular, they are quite sensitive to the choice of microturbulent velocities. These effects could produce an increase in the uncertainties.

The $s$-process elements can be considered as a signature of the processes that occur in intermediate-mass AGB stars (Busso et al. 2001), whose wind could have polluted the primordial material from which the second generation of stars formed. If the spread in $s$-process contents in NGC 4372 is real, this would argue against the abundances of $s$-process elements being intrinsic to the cluster and suggest that AGB stars can be possible polluters; however, none of the analyzed heavy elements show any clear relations with other lighter elements that could suggest any obvious pollution by AGB stars. Since this potential spread seems unrelated to the spreads in light elements, the variations could be attributed to the original inhomogeneities in the gas that formed the cluster. A larger sample survey of stars at various evolutionary phases and extensive abundance analysis of a range of key nucleosynthetic indicators would be essential to confirm whether this potential star-to-star scatter is real.

\section{Summary}

In this paper we present the first detailed chemical abundances of 14 elements in seven red giant members of NGC 4372 using high-resolution, high signal-to-noise spectroscopy. Chemical abundances have been computed by Concepcion Node within the GES collaboration. The classical EW method was used when possible. For five elements whose lines are affected by blending, 
the spectrum-synthesis method was preferred. One of the stars in our sample shows a radial velocity that agrees with the cluster bulk but a distinct chemical signature, so we excluded it from the statistical analysis.

We found a metallicity of $[\mathrm{Fe} / \mathrm{H}]=-2.19 \pm 0.02$ with $\sigma_{\text {obs }}=$ 0.03 dex, in good agreement with previous, low-resolution, studies. We ruled out an intrinsic metallicity spread, although the low value for the one (excluded) star should be born in mind. We confirmed the Na-O anticorrelation although it was not very extended, probably owing to our small sample. The abundances of $\mathrm{O}$ are relatively high compared with other globular clusters, which could indicate that NGC 4372 was formed in an environment with high $\mathrm{O}$ for its metallicity. Intrinsic spreads were also seen in other light elements; in particular, an apparent $\mathrm{Mg}$ $\mathrm{Al}$ anticorrelation was detected. The Fe-peak elements generally show good agreement with other GCs and halo field stars with no dispersion. The $\alpha$ elements show an enhancement of $[\alpha / \mathrm{Fe}]=$ $+0.37 \pm 0.07$ that is typical of other GCs and that indicates similar fast star formation time scales. A relation between light and heavy $s$-process elements was identified. A larger sample of stars at various evolutionary phases and extensive chemical analysis is required for a more definitive analysis.

Acknowledgements. We thank Michele Bellazzini for a very careful reading and useful comments and suggestions that helped to improve the quality of the paper. We also thank the anonymous referee for comments that greatly improved this paper. Based on data products from observations made with ESO Telescopes at the La Silla Paranal Observatory under program ID 188.B3002. These data products have been processed by the Cambridge Astronomy Survey Unit (CASU) at the Institute of Astronomy, University of Cambridge, and by the FLAMES/UVES reduction team at INAF/Osservatorio Astrofisico di Arcetri. These data have been obtained from the Gaia-ESO Survey Data Archive, prepared and hosted by the Wide Field Astronomy Unit, Institute for Astronomy, University of Edinburgh, which is funded by the UK Science and Technology Facilities Council. This work was partly supported by the European Union FP7 program through ERC grant number 320360 and by the Leverhulme Trust through grant RPG-2012-541. We acknowledge the support from INAF and Ministero dell' Istruzione, dell' Università' e della Ricerca (MIUR) in the form of the grant "Premiale VLT 2012". The results presented here benefit from discussions held during the Gaia-ESO workshops and conferences supported by the ESF (European Science Foundation) through the GREAT Research Network Program. I.S.R. gratefully acknowledges the support provided by the Gemini-CONICYT project 32110029. C.M. acknowledges the support from CONICYT-PCHA/Doctorado Nacional/2014-21141057. D.G. gratefully acknowledges support from the Chilean BASAL Centro de Excelencia en Astrofísica y Tecnologías Afines (CATA) gran PFB-06/2007. S.V. gratefully acknowledges the support provided by Fondecyt reg. 1130721. A.K. and N.K. acknowledge the Deutsche Forschungsgemeinschaft for funding from EmmyNoether grant Ko 4161/1. U.H. acknowledges support from the Swedish National Space Board (SNSB). S.G.S acknowledges the support from the Fundação para a Ciência e Tecnologia, FCT (Portugal) and POPH/FSE (EC), in the form of the fellowships SFRH/BPD/47611/2008.

\section{References}

Alcaino, G. 1974, A\&AS, 13, 345

Alcaino, G., Liller, W., Alvarado, F., \& Wenderoth, E. 1991, AJ, 102, 159

Arlandini, C., Käppeler, F., Wisshak, K., et al. 1999, ApJ, 525, 886

Arnould, M., Goriely, S., \& Jorissen, A. 1999, A\&A, 347, 572

Bastian, N., Lamers, H. J. G. L. M., de Mink, S. E., et al. 2013, MNRAS, 436, 2398

Bragaglia, A., Carretta, E., Gratton, R. G., et al. 2010, ApJ, 720, L41

Bragaglia, A., Sneden, C., Carretta, E., et al. 2014, ApJ, 796, 68

Bressan, A., Marigo, P., Girardi, L., et al. 2012, MNRAS, 427, 127

Brocato, E., Buonanno, R., Malakhova, Y., \& Piersimoni, A. M. 1996, A\&A, 311,778

Busso, M., Gallino, R., Lambert, D. L., Travaglio, C., \& Smith, V. V. 2001, ApJ, 557,802

Caloi, V., \& D'Antona, F. 2011, MNRAS, 417, 228

Carretta, E. 2006, AJ, 131, 1766

Carretta, E., \& Gratton, R. G. 1997, A\&AS, 121, 95

Carretta, E., Bragaglia, A., Gratton, R., \& Lucatello, S. 2009a, A\&A, 505, 139
Carretta, E., Bragaglia, A., Gratton, R. G., et al. 2009b, A\&A, 505, 117 Carretta, E., Bragaglia, A., Gratton, R., D’Orazi, V., \& Lucatello, S. 2009c, A\&A, 508, 695

Carretta, E., Bragaglia, A., Gratton, R., et al. 2010a, ApJ, 712, L21

Carretta, E., Bragaglia, A., Gratton, R. G., et al. 2010b, A\&A, 520, A95

Carretta, E., Bragaglia, A., Gratton, R. G., et al. 2010c, A\&A, 516, A55

Carretta, E., Lucatello, S., Gratton, R. G., Bragaglia, A., \& D’Orazi, V. 2011, A\&A, 533, A69

Carretta, E., Bragaglia, A., Gratton, R. G., et al. 2014, A\&A, 561, A87

Casetti-Dinescu, D. I., Girard, T. M., Herrera, D., et al. 2007, AJ, 134, 195

Cayrel, R., Depagne, E., Spite, M., et al. 2004, A\&A, 416, 1117

Cohen, J. G. 2004, AJ, 127, 1545

Cohen, J. G., Kirby, E. N., Simon, J. D., \& Geha, M. 2010, ApJ, 725, 288

Cunha, K., Smith, V. V., Johnson, J. A., et al. 2015, ApJ, 798, L41

D’Antona, F., Caloi, V., Montalbán, J., Ventura, P., \& Gratton, R. 2002, A\&A, 395, 69

de Laverny, P., Recio-Blanco, A., Worley, C. C., \& Plez, B. 2012, A\&A, 544, A126

de Mink, S. E., Pols, O. R., Langer, N., \& Izzard, R. G. 2009, A\&A, 507, L1

Decressin, T., Meynet, G., Charbonnel, C., Prantzos, N., \& Ekström, S. 2007, A\&A, 464, 1029

Denissenkov, P. A., Da Costa, G. S., Norris, J. E., \& Weiss, A. 1998, A\&A, 333, 926

D’Ercole, A., Vesperini, E., D’Antona, F., McMillan, S. L. W., \& Recchi, S. 2008, MNRAS, 391, 825

Ferraro, F. R., Dalessandro, E., Mucciarelli, A., et al. 2009, Nature, 462, 483

Fulbright, J. P. 2000, AJ, 120, 1841

Fulbright, J. P. 2002, AJ, 123, 404

Geisler, D., Piatti, A. E., Claria, J. J., \& Minniti, D. 1995, AJ, 109, 605

Geisler, D., Wallerstein, G., Smith, V. V., \& Casetti-Dinescu, D. I. 2007, PASP, 119,939

Geisler, D., Villanova, S., Carraro, G., et al. 2012, ApJ, 756, L40

Gilmore, G., Randich, S., Asplund, M., et al. 2012, The Messenger, 147, 25

Gratton, R. G., \& Sneden, C. 1994, A\&A, 287, 927

Gratton, R. G., Bonifacio, P., Bragaglia, A., et al. 2001, A\&A, 369, 87

Gratton, R., Sneden, C., \& Carretta, E. 2004, ARA\&A, 42, 385

Gratton, R. G., Carretta, E., \& Bragaglia, A. 2012, A\&ARv, 20, 50

Grevesse, N., Asplund, M., \& Sauval, A. J. 2007, Space Sci. Rev., 130, 105

Gustafsson, B., Edvardsson, B., Eriksson, K., et al. 2008, A\&A, 486, 951

Harris, W. E. 1996, AJ, 112, 1487

Hartwick, F. D. A., \& Hesser, J. E. 1973, ApJ, 186, 1171

Ishigaki, M. N., Aoki, W., Arimoto, N., \& Okamoto, S. 2014, A\&A, 562, A146

Ivans, I. I., Kraft, R. P., Sneden, C., et al. 2001, AJ, 122, 1438

Joo, S.-J., \& Lee, Y.-W. 2013, ApJ, 762, 36

Kacharov, N., Koch, A., \& McWilliam, A. 2013, A\&A, 554, A81

Kacharov, N., Bianchini, P., Koch, A., et al. 2014, A\&A, 567, A69

Koch, A., \& McWilliam, A. 2011, AJ, 142, 63

Koch, A., Grebel, E. K., Gilmore, G. F., et al. 2008a, AJ, 135, 1580

Koch, A., McWilliam, A., Grebel, E. K., Zucker, D. B., \& Belokurov, V. 2008b, ApJ, 688, L13

Koch, A., Côté, P., \& McWilliam, A. 2009, A\&A, 506, 729

Langer, G. E., \& Hoffman, R. D. 1995, PASP, 107, 1177

Lapenna, E., Mucciarelli, A., Lanzoni, B., et al. 2014, ApJ, 797, 124

Lardo, C., Pancino, E., Bellazzini, M., et al. 2013, MNRAS, 433, 1941

Lardo, C., Pancino, E., Bellazzini, M., et al. 2015, A\&A, 573, A115

Lee, J.-W., \& Carney, B. W. 2002, AJ, 124, 1511

Lee, J.-W., Carney, B. W., \& Habgood, M. J. 2005, AJ, 129, 251

Letarte, B., Hill, V., Jablonka, P., et al. 2006, A\&A, 453, 547

Marino, A. F., Villanova, S., Piotto, G., et al. 2008, A\&A, 490, 625

Marino, A. F., Milone, A. P., Piotto, G., et al. 2009, A\&A, 505, 1099

Marino, A. F., Pancino, E., Mucciarelli, A., et al. 2011, ApJ, 731, 64

Mashonkina, L. I., Shimanskii, V. V., \& Sakhibullin, N. A. 2000, Astron. Rep., 44, 790

Masseron, T. 2006, Ph.D. Thesis, Observatoire de Paris

McLean, B. J., Greene, G. R., Lattanzi, M. G., \& Pirenne, B. 2000, in Astronomical Data Analysis Software and Systems IX, eds. N. Manset, C. Veillet, \& D. Crabtree, ASP Conf. Ser., 216, 145

Modigliani, A., \& Larsen, J. M. 2012, FLAMES-UVES pipeline user manual, 14th edn. (ESO)

Modigliani, A., Mulas, G., Porceddu, I., et al. 2004, The Messenger, 118, 8

Muñoz, C., Geisler, D., \& Villanova, S. 2013, MNRAS, 433, 2006

Mucciarelli, A., Origlia, L., Ferraro, F. R., \& Pancino, E. 2009, ApJ, 695, L134

Mucciarelli, A., Pancino, E., Lovisi, L., Ferraro, F. R., \& Lapenna, E. 2013, ApJ, 766, 78

Mucciarelli, A., Dalessandro, E., Ferraro, F. R., Origlia, L., \& Lanzoni, B. 2014, ApJ, 793, L6

Pancino, E., \& Gaia-ESO Survey consortium, O. B. O. T. 2012, ArXiv e-print [arXiv: 1206.6291] 
Pignatari, M., Gallino, R., Heil, M., et al. 2010, ApJ, 710, 1557 Piotto, G., Bedin, L. R., Anderson, J., et al. 2007, ApJ, 661, L53 Randich, S., \& Gilmore, G. 2012, in Science from the Next Generation Imaging and Spectroscopic Surveys

Reddy, B. E., Tomkin, J., Lambert, D. L., \& Allende Prieto, C. 2003, MNRAS, 340,304

Reddy, B. E., Lambert, D. L., \& Allende Prieto, C. 2006, MNRAS, 367, 1329 Roederer, I. U. 2011, ApJ, 732, L17

Roederer, I. U., Cowan, J. J., Karakas, A. I. et al. 2010, ApJ, 724, 975

Rosenberg, A., Piotto, G., Saviane, I., \& Aparicio, A. 2000, A\&AS, 144, 5 Rutledge, G. A., Hesser, J. E., \& Stetson, P. B. 1997, PASP, 109, 907

Sacco, G. G., Morbidelli, L., Franciosini, E., et al. 2014, A\&A, 565, A113

Sbordone, L., Bonifacio, P., Marconi, G., Buonanno, R., \& Zaggia, S. 2005, A\&A, 437, 905

Sbordone, L., Bonifacio, P., Buonanno, R., et al. 2007, A\&A, 465, 815

Schlegel, D. J., Finkbeiner, D. P., \& Davis, M. 1998, ApJ, 500, 525

Shetrone, M. D., Côté, P., \& Sargent, W. L. W. 2001, ApJ, 548, 592

Simmerer, J., Sneden, C., Cowan, J. J., et al. 2004, ApJ, 617, 1091

Skrutskie, M. F., Cutri, R. M., Stiening, R., et al. 2006, AJ, 131, 1163

Smiljanic, R., Korn, A. J., Bergemann, M., et al. 2014, A\&A, 570, A122

Sneden, C. A. 1973, Ph.D. Thesis, The University of Texas at Austin

Sneden, C., Kraft, R. P., Shetrone, M. D., et al. 1997, AJ, 114, 1964
Sneden, C., Johnson, J., Kraft, R. P., et al. 2000, ApJ, 536, L85

Stetson, P. B. 1987, PASP, 99, 191

Stetson, P. B. 1992, in Astronomical Data Analysis Software and Systems I, eds.

D. M. Worrall, C. Biemesderfer, \& J. Barnes, ASP Conf. Ser., 25, 297

Stetson, P. B. 2000, PASP, 112, 925

Stetson, P. B., \& Pancino, E. 2008, PASP, 120, 1332

Tautvaišienè, G., Wallerstein, G., Geisler, D., Gonzalez, G., \& Charbonnel, C. 2004, AJ, 127, 373

Thygesen, A. O., Sbordone, L., Andrievsky, S., et al. 2014, A\&A, 572, A108

Valdes, F. G. 1998, in Astronomical Data Analysis Software and Systems VII, eds. R. Albrecht, R. N. Hook, \& H. A. Bushouse, ASP Conf. Ser., 145, 53

Ventura, P., D’Antona, F., Mazzitelli, I., \& Gratton, R. 2001, ApJ, 550, L65

Villanova, S., \& Geisler, D. 2011, A\&A, 535, A31

Villanova, S., Geisler, D., Carraro, G., Moni Bidin, C., \& Muñoz, C. 2013, ApJ, 778,186

Worley, C. C., Hill, V., Sobeck, J., \& Carretta, E. 2013, A\&A, 553, A47

Yong, D., \& Grundahl, F. 2008, ApJ, 672, L29

Yong, D., Grundahl, F., Nissen, P. E., Jensen, H. R., \& Lambert, D. L. 2005, A\&A, 438, 875

Yong, D., Roederer, I. U., Grundahl, F., et al. 2014, MNRAS, 441, 3396

Zacharias, N., Finch, C., Girard, T., et al. 2010, AJ, 139, 2184

Zinn, R., \& West, M. J. 1984, ApJS, 55, 45 